\title{
Variance Error Quantifications That Are Exact for Finite-Model Order
}

\author{
Brett Ninness and Håkan Hjalmarsson
}

\begin{abstract}
This paper is concerned with the frequency domain quantification of noise induced errors in dynamic system estimates. Preceding and seminal work on this problem has suggested general expressions that are approximations whose accuracy increases with observed data length and model order. In the interests of improved accuracy, this paper provides new expressions whose accuracy depends only on data length. They are therefore "exact" for arbitrarily small true model order. Other authors have recognized the importance of such expressions and have derived them for the case of finite-impulse response-like model structures in which denominators are fixed at true values and only numerator terms are estimated. This paper progresses beyond this situation to address the more general output-error and Box-Jenkins structures in which full dynamics models (both numerator and denominator terms) and noise models may be estimated. A key aspect of the work here is that it establishes that the variance quantification problem is equivalent to that of deriving the reproducing kernel for a subspace that depends on the model structure being employed.
\end{abstract}

Index Terms-Orthonormal bases, parameter estimation, system identification, vaiance error.

\section{INTRODUCTION}

W JHEN identifying a system model on the basis of observed data, it is essential to quantify the likely error in that estimated model. Typically, this consists of two components. The first, a so-called "bias error," is the result of the model structure being less complex than the system being estimated. The second, called "variance error," is caused by corruption of the input-output data measurements.

Furthermore, when the corruption can be modeled as an additive stochastic process, and the underlying system is linear, then it is arguable that the total error in any identified model that passes a validation test is dominated by variance error [1].

In this common case, or when the model structure is rich enough to encompass the true underlying dynamics, the quantification of the total estimation error then becomes a question of assessing variance error. In relation to this, if the widely used prediction-error method with a quadratic criterion is employed [2], [3], then a seminal result is that under open-loop conditions

Manuscript received May 23, 2003; revised December 4, 2003 and April 2, 2004. Recommended by Associate Editor E. Bai. This work was supported by the Australian Research Council. Portions of this work were completed while the authors were visiting S3-Automatic Control, The Royal Institute of Technology, Stockholm, Sweden.

B. Ninness is with the School of Electrical Engineering and Computer Science, the University of Newcastle, Newcastle, Australia (e-mail: brett@ee.newcastle.edu.au).

H. Hjalmarsson is with the Department of Sensors, Signals and Systems (Automatic Control), The Royal Institute of Technology, S-100 44 Stockholm, Sweden (e-mail: hakan.hjalmarsson@s3.e.kth.se).

Digital Object Identifier 10.1109/TAC.2004.832202 the noise-induced error, as measured by the variability of the ensuing frequency response estimate $G\left(e^{j \omega}, \hat{\theta}_{N}^{n}\right)$, obeys [4], [5], $[2],[6]$

$$
\lim _{m \rightarrow \infty} \lim _{N \rightarrow \infty} \frac{N}{m} \operatorname{Var}\left\{G\left(e^{j \omega}, \hat{\theta}_{N}^{n}\right)\right\}=\frac{\Phi_{\nu}(\omega)}{\Phi_{u}(\omega)} .
$$

Here, $\Phi_{\nu}$ and $\Phi_{u}$ are, respectively, the measurement noise and input excitation power spectral densities, and $\hat{\theta}_{N}^{n}$ is the prediction error estimate based on $N$ observed data points of a vector $\theta^{n} \in \mathbf{R}^{n}$ that parameterises a model structure $G\left(q, \theta^{n}\right)$ for which (essentially) the model order $m=\operatorname{dim} \theta^{n} /\left(2^{d}\right)$ where $d$ is the number of denominator polynomials to be estimated in the model structure.

Although this result is asymptotic in both data length $N$ and model order $m$, it suggests the very well-known approximation for finite data and model order of

$$
\operatorname{Var}\left\{G\left(e^{j \omega}, \hat{\theta}_{N}^{n}\right)\right\} \approx \frac{m}{N} \frac{\Phi_{\nu}(\omega)}{\Phi_{u}(\omega)} .
$$

Apart from its simplicity, a key factor underlying the importance and popularity of the quantification (2) is that, according to its derivation [4], [5], [2], [6], the expression (1) applies for a very wide class of so-called 'shift invariant' model structures. For example, all the well known finite-impulse response (FIR), ARX, ARMAX, output-error, and Box-Jenkins structures are shift invariant [4]. Additionally, as shown in [2], the result (1) also applies when nonparametric (spectral based) estimation methods [7], [2] are employed provided that the $m$ term in (2) is replaced by one dependent on the number of data points (and the windowing function) used.

However, a fundamental aspect of the approximation (2) is that (since it is derived from (1) which is asymptotic in model order $m$ ) its accuracy for realistic finite model orders is not guaranteed [8], [9].

In relation to this, [10] has also recognized the importance of this question by choosing to address the specific case of a model structure in which only a polynomial numerator term $B\left(q, \theta^{n}\right)$ is estimated, while the denominator is fixed according to the $m_{a}$ true underlying pole positions, and a fixed but arbitrary moving average (MA) noise model is included. With these caveats, together with further ones that the input is an autoregressive order $m_{f}\left(\mathrm{AR}\left(m_{f}\right)\right)$ process, and that the numerator order is $m_{b}=m_{a}+m_{f}$ so that it overmodels the underlying true dynamics, then the work [10] establishes the following result:

$$
\lim _{N \rightarrow \infty} N \cdot \operatorname{Var}\left\{G\left(e^{j \omega}, \hat{\theta}_{N}^{n}\right)\right\}=\frac{\Phi_{\nu}(\omega)}{\Phi_{u}(\omega)} \sum_{k=1}^{m_{a}+m_{f}} \frac{1-\left|\xi_{k}\right|^{2}}{\left|e^{j \omega}-\xi_{k}\right|^{2}} .
$$


Here, the $\left\{\xi_{k}\right\}$ are the combined poles of the true underlying dynamics, and the $\operatorname{AR}\left(m_{f}\right)$ process which is the observed input. Furthermore, the expression (3) assumes that the true and fixed MA noise models are equal, although the full work in [10] does consider more general cases; as will be discussed in more detail in Section V-B.

More importantly though, the expression (3) is exact for finite-model order $m_{b}=m_{a}+m_{f}$, and therefore it is clear, as observed in [10], that the associated quantification

$$
\operatorname{Var}\left\{G\left(e^{j \omega}, \hat{\theta}_{N}^{n}\right)\right\} \approx \frac{1}{N} \frac{\Phi_{\nu}(\omega)}{\Phi_{u}(\omega)} \sum_{k=1}^{m_{a}+m_{f}} \frac{1-\left|\xi_{k}\right|^{2}}{\left|e^{j \omega}-\xi_{k}\right|^{2}}
$$

should be more accurate than (2), and this is demonstrated empirically in [10].

Furthermore, again as observed in [10], the quantification (4) is significantly different to the widely used pre-existing one (2) in that a frequency independent term of $m=m_{a}+m_{f}$ in (2) is replaced by a frequency dependent sum over $m_{a}+m_{f}$ components in (4), which can clearly exhibit orders of magnitude variation as $\omega$ varies.

Therefore, although it is reasonable to expect that the expression (2) is a compromise that sacrifices accuracy for simplicity, in some cases this tradeoff can lead to very misleading expressions and, hence, [10] establishes that further study is clearly warranted.

With this pre-existing work [10] as motivation, the work here extends the results of it and related contributions [11]-[14] from the fixed denominator FIR-like model class considered there to the much more general situations of output-error and Box-Jenkins modeling. In all cases, and in the interests of accuracy, expressions are derived here that hold exactly for finite model orders.

A key conclusion arising from these new results is that, while the recent work [10] suggests that when substantial errors occur in the quantification (2) they are due to special factors such as 'erroneous noise models, colored inputs and fixed poles', the new expressions developed here establish that these substantial errors in (2) are a much more general phenomenon that are not predicated on these special factors.

For example, reference [15], which builds on the work here, establishes that the convergence result (1) underpinning (2) is dominated by a choice of a regularization point which is completely arbitrary, and it is only the specific regularization choice that fixes excess pole/zero cancellations at the origin that leads to (2). Other choices can lead to results arbitrarily different to (1) and (2) and, hence, it is rather problematic to draw conclusions about fundamental aspects of an estimation problem from approximate quantifications such as (2).

Underpinning the work here is a new approach to the variance quantification problem that involves the use of what is known as a "reproducing kernel." This kernel is quantified via a particular orthonormal basis which has been used in previous works [10], [14]. However, it was employed there in a manner that is fundamentally different to that used in this paper. Here, via the reproducing kernel interpretation, a geometric interpretation of the variance quantification problem is exposed. This minimizes the arithmetic required to derive variance quantifications, while also allowing for exact quantifications not provided in previous works.

This paper now proceeds in Section II to provide a very brief simulation example that illustrates the need for the new analysis pursued here by establishing how misleading the quantification (2) may be. The following Section III then embarks on a discussion of the reproducing kernel ideas underpinning this work, and establishes several key technical results, while Section IV establishes the model structures, estimation method and data collection scenarios that are addressed here.

The main results of the paper are provided in Section V, followed by a concluding section which reinforces certain issues and points to related papers that build on the work presented here.

\section{Motivation}

In the interests of illustrating the practical (and theoretical) significance of the analysis undertaken here, we provide a brief illustrative simulation example in which the following simple system:

$$
G(q)=\frac{0.05}{q-0.95}
$$

is used to generate an $N=10000$ sample input-output data record where the output $\left\{y_{t}\right\}$ is corrupted by white Gaussian noise of variance $\sigma^{2}=10$, and where the input $\left\{u_{t}\right\}$ is a realization of a stationary, zero mean, unit variance white Gaussian process.

On the basis of this observed data, a first-order output-error model structure $G\left(q, \hat{\theta}_{N}^{n}\right)$ is estimated, and the sample mean square error in this estimate over 1000 estimation experiments with different input and noise realizations is used as an estimate of $\operatorname{Var}\left\{G\left(e^{j \omega}, \hat{\theta}_{N}^{n}\right)\right\}$ which is plotted as a solid line in Fig. 1(a).

The "classical" approximation (2) is shown as the flat and straight dash-dot line in that same figure, and is clearly a poor approximation to the true variability. For example, it is qualitatively misleading by not eliciting the "low pass" nature of $\operatorname{Var}\left\{G\left(e^{j \omega}, \hat{\theta}_{N}^{n}\right)\right\}$, and instead suggesting that it is "all pass."

More importantly though, it is quantitatively misleading in a rather dramatic way, in that it is inaccurate at low and high frequencies by orders of magnitude. If it were used to perform the very common procedure of judging the radius of error bounds on estimated Nyquist plots, then those radii would be approximately one hundred times too small near zero frequency, and more than ten times too large at the folding frequency.

In recognition of this, a main contribution of this paper is to establish that under certain assumptions on the input and measurement noise, then in the output-error case considered in this section

$$
\lim _{N \rightarrow \infty} N \cdot \operatorname{Var}\left\{G\left(e^{j \omega}, \hat{\theta}_{N}^{n}\right)\right\}=2 \cdot \frac{\Phi_{\nu}(\omega)}{\Phi_{u}(\omega)} \sum_{k=1}^{m} \frac{1-\left|\xi_{k}\right|^{2}}{\left|e^{j \omega}-\xi_{k}\right|^{2}}
$$

where the $\left\{\xi_{k}\right\}$ are the poles of the underlying true system (i.e., the input-output dynamics). Note that, like the recent results in [10], where more restrictive model structures with fixed denominator were considered, the expression (6) also applies 


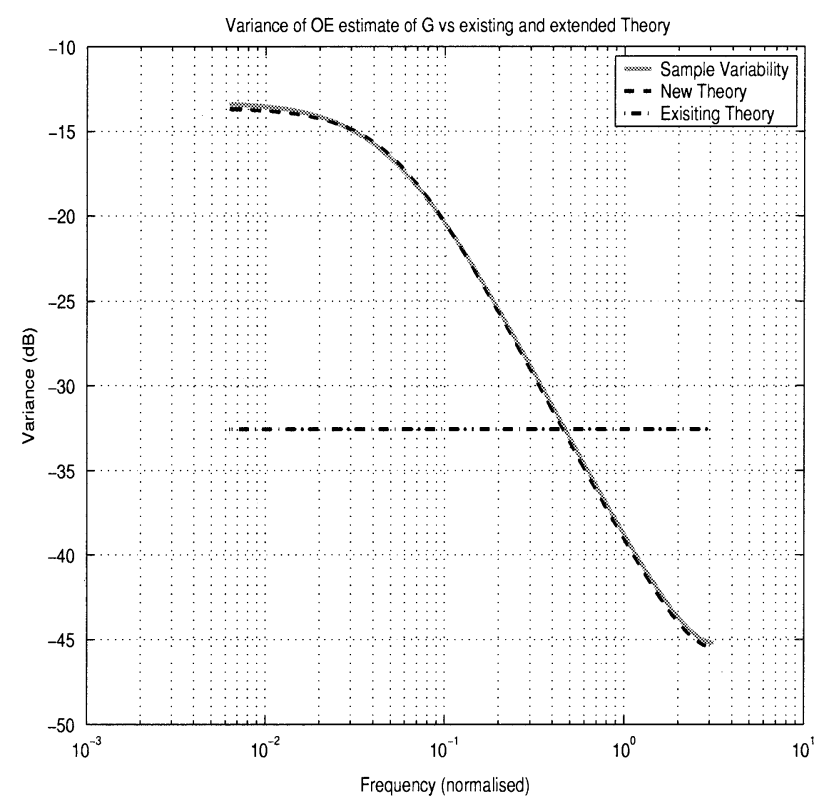

(a) True Variability vs. Theory

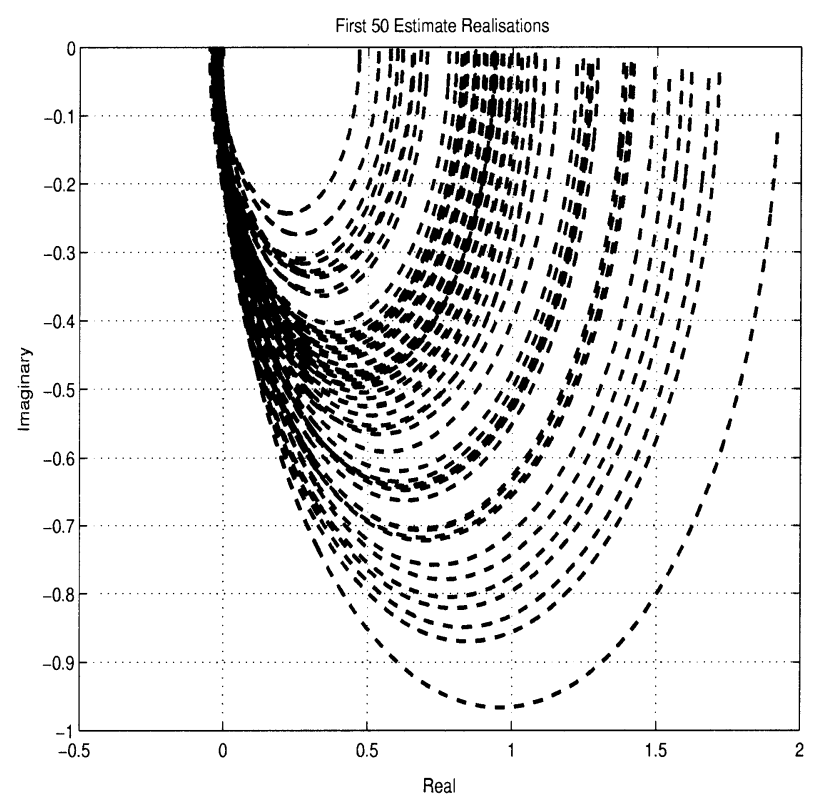

(b) Sample Estimate Realisations

Fig. 1. Variability of output-error estimates. (a) True variability versus theoretically derived approximations. The solid line is the Monte Carlo estimate of the true variability, the dash-dot line is the pre-existing approximation (2) which does not account for system poles or model structure. The dashed line is the new approximation presented in (6) whereby estimated system pole positions $\left\{\xi_{1}, \ldots, \xi_{m}\right\}$ and the fact that an output-error structure is employed are both accounted for. (b) First 50 (of 1000) estimate realizations to give a sense of the scale of the variability being quantified in (a).

for finite-model order $m$ and, hence, the ensuing approximation suggested in this paper of

$$
\operatorname{Var}\left\{G\left(e^{j \omega}, \hat{\theta}_{N}^{n}\right)\right\} \approx \frac{2}{N} \cdot \frac{\Phi_{\nu}(\omega)}{\Phi_{u}(\omega)} \sum_{k=1}^{m} \frac{1-\left|\xi_{k}\right|^{2}}{\left|e^{j \omega}-\xi_{k}\right|^{2}}
$$

does not depend on the model order $m$ being large. This is in contrast to the work underlying the well-known pre-existing approximation (2).

In a sense then, the right-hand side of (7) is "exact" for finite model order $m$, as illustrated by the dashed line in Fig. 1(a) which is the expression (7), and exactly matches the true variability shown as the solid line. Therefore, in the sequel, when referring to an "exact quantification of variance," it will be to avoid more cumbersome (but more accurate) descriptions such as "exact quantification of the asymptotic in $N$ variance." The point to be made is that expressions such as (7) to be derived here will not depend on the model order being large.

Finally, Fig. 1(b) illustrates the first 50 (of 1,000) estimate realizations (represented via the corresponding Nyquist plot of $\left.G\left(e^{j \omega}, \hat{\theta}_{N}^{n}\right)\right)$ that are averaged to produce an estimate of the true variability shown as the solid line in Fig. 1(a). This is shown to emphasise the possible large scale of the error that can be accurately quantified by the results of this paper. That is, the results here are not restricted to the evaluation of minor effects.

\section{TECHNICAL PRELIMINARIES}

In this paper, the idea of what is called a "reproducing kernel" for a space will prove to be a vital tool that allows for the direct simplification of complicated quantities via what is essentially a geometric principle. In presenting these underlying ideas, the following notation will be employed.

- C, R, T: Respectively, the fields of complex and real numbers, and the complex unit circle $\{z \in \mathbf{C}:|z|=1\}$.

- $x^{\star}, x^{-\star}$ : Respectively, for arbitrary $x$, the complex conjugate transpose and the inverse of the complex conjugate transpose.

- $[x]_{k}$ : The $k$ 'th element of a vector $x$, or the $k$ 'th row of a matrix $x$.

- $\langle f, g\rangle$ : The inner product, defined for arbitrary functions $f, g:[-\pi, \pi] \rightarrow \mathbf{C}^{p}$ by

$$
\langle f, g\rangle=\frac{1}{2 \pi} \int_{-\pi}^{\pi} g^{\star}(\lambda) f(\lambda) \mathrm{d} \lambda .
$$

- $L_{2}, L_{2}([-\pi, \pi])$ : The space of functions $f:[-\pi, \pi] \rightarrow \mathbf{C}^{p}$ such that

$$
\|f\|^{2} \triangleq\langle f, f\rangle<\infty
$$

- $\gamma(z)$ : The positive real part of a function, say $H(z) H(1 / z)$, which if the latter has Laurent expansion

$$
H(z) H(1 / z)=\sum_{k=-\infty}^{\infty} c_{k} z^{k}
$$

is defined as

$$
\gamma(z) \triangleq \frac{c_{0}}{2}+\sum_{k=1}^{\infty} c_{k} z^{-k}
$$

so that $\gamma(z)$ is analytic on $|z|>1$ and $H(z) H(1 / z)=$ $\gamma(z)+\gamma(1 / z)$. 


\section{A. Reproducing Kernels}

Consider a subspace $X_{n}$ of $L_{2}$ defined by a sequence $\left\{g_{k}\right\}$ of elements of $L_{2}$ as $\left(g_{k}:[-\pi, \pi] \rightarrow \mathbf{C}^{p}\right)$

$$
X_{n} \triangleq \operatorname{Span}\left\{g_{1}, \ldots, g_{n}\right\} \text {. }
$$

The so-called "reproducing kernel" $\varphi_{n}(\lambda, \omega):[-\pi, \pi] \times$ $[-\pi, \pi] \rightarrow \mathbf{C}^{p \times p}$ for this space $X_{n}$ of $\mathbf{C}^{p}$ valued functions is an entity such that for any $\alpha \in \mathbf{C}^{p}$ [16], [17]

$$
\varphi_{n}(\cdot, \omega) \alpha \in X_{n} \quad \forall \omega \in[-\pi, \pi]
$$

and for any $f \in X_{n}$

$$
\left\langle f(\cdot), \varphi_{n}(\cdot, \omega) \alpha\right\rangle=\alpha^{\star} f(\omega) .
$$

Here and in the sequel, for inner product expressions like (14), the implied integration according to (8) will be over the common argument (in the aforementioned case indicated by $\cdot$ ).

Since the mapping $f \mapsto f(\omega)$ is a (bounded) linear functional $X_{n} \rightarrow \mathbf{C}^{p}$, then it is a consequence of the Riesz Representation Theorem [18] that a function $\varphi_{n}(\lambda, \omega)$ satisfying (13) and (14) exists. Furthermore, $\varphi_{n}(\lambda, \omega)$ is "Hermitian symmetric" in that for any $\alpha, \beta \in \mathbf{C}^{p}$

$$
\begin{aligned}
\alpha^{\star} \varphi_{n}(\mu, \omega) \beta & =\left\langle\varphi_{n}(\lambda, \omega) \beta, \varphi_{n}(\lambda, \mu) \alpha\right\rangle \\
& =\overline{\left\langle\varphi_{n}(\lambda, \mu) \alpha, \varphi_{n}(\lambda, \omega) \beta\right\rangle}=\overline{\beta^{\star} \varphi_{n}(\omega, \mu) \alpha} .
\end{aligned}
$$

This implies that $\varphi_{n}(\lambda, \omega)$ is the unique element that has the property (14) and also satisfies $\varphi_{m}(\lambda, \omega) \alpha \in X_{n}$ for any $\alpha \in$ $\mathbf{C}^{p}$, since if another function $\gamma_{n}(\lambda, \omega)$ also had these properties then it would hold that for arbitrary $\alpha, \beta \in \mathbf{C}^{p}$

$$
\begin{aligned}
\alpha^{\star} \gamma_{n}(\mu, \omega) \beta & =\left\langle\gamma_{n}(\lambda, \omega) \beta, \varphi_{n}(\lambda, \mu) \alpha\right\rangle \\
& =\overline{\left\langle\varphi_{n}(\lambda, \mu) \alpha, \gamma_{n}(\lambda, \omega) \beta\right\rangle} \\
& =\overline{\beta^{\star} \varphi_{n}(\omega, \mu) \alpha}=\alpha^{\star} \varphi_{n}(\mu, \omega) \beta .
\end{aligned}
$$

This last uniqueness property will be particularly vital in later developments. Despite this fact, there may (of course) be several different ways of expressing the unique reproducing kernel, two of which are particularly important in later developments.

Lemma 3.1 (Expressions for the Reproducing Kernel): Consider the subspace $X_{n} \subseteq L_{2}$ defined via (12) with alternative basis $\left\{\mathcal{B}_{k}\right\}$

$$
X_{n}=\operatorname{Span}\left\{\mathcal{B}_{1}(\lambda), \ldots, \mathcal{B}_{n}(\lambda)\right\}
$$

which is orthonormal in that $\left\langle\mathcal{B}_{k}, \mathcal{B}_{\ell}\right\rangle=\delta(k-\ell)$ where the latter is the Kronecker delta function. Then the reproducing kernel $\varphi_{n}(\lambda, \omega)$ on $X_{n}$ may be expressed as

$$
\varphi_{n}(\lambda, \omega)=\sum_{k=1}^{n} \mathcal{B}_{k}(\lambda) \mathcal{B}_{k}^{\star}(\omega) .
$$

Furthermore, define the matrix valued function $\Psi:[-\pi, \pi] \rightarrow$ $\mathbf{C}^{n \times p}$ and the matrix $T_{n} \in \mathbf{R}^{n \times n}$ according to

$$
\begin{aligned}
\Psi(\lambda) & \triangleq\left[g_{1}(\lambda), \ldots, g_{n}(\lambda)\right]^{T} \\
T_{n} & \triangleq \frac{1}{2 \pi} \int_{-\pi}^{\pi} \overline{\Psi(\lambda)} \Psi^{T}(\lambda) d \lambda .
\end{aligned}
$$

Then, the reproducing kernel $\varphi_{n}(\lambda, \omega)$ on $X_{n}$ may also be expressed as

$$
\varphi_{n}(\lambda, \omega)=\Psi^{T}(\lambda) T_{n}^{-1} \overline{\Psi(\omega)}
$$

Proof: To prove (17) we proceed as follows. Suppose that $f(\lambda)=\sum_{\tau} c_{\tau} \mathcal{B}_{\tau}(\lambda)$ for some constants $\left\{c_{\tau}\right\}$. Then, for $\alpha \in$ $\mathrm{C}^{p}$ arbitrary

$$
\begin{aligned}
& \left\langle\sum_{\tau=1}^{n} c_{\tau} \mathcal{B}_{\tau}(\lambda), \sum_{k=1}^{n} \mathcal{B}_{k}(\lambda) \mathcal{B}_{k}^{\star}(\omega) \alpha\right\rangle \\
& =\sum_{\tau=1}^{n} c_{\tau} \sum_{k=1}^{n} \alpha^{\star} \mathcal{B}_{k}(\omega)\left\langle\mathcal{B}_{\tau}(\lambda), \mathcal{B}_{k}(\lambda)\right\rangle \\
& =\sum_{\tau=1}^{n} c_{\tau} \alpha^{\star} \mathcal{B}_{\tau}(\omega)=\alpha^{\star} f(\omega)
\end{aligned}
$$

which establishes (17). To prove (19), denote by $e_{k} \in \mathbf{C}^{n}$ the vector of all zeros save for a 1 in the $k$ 'th position so that $g_{k}(\lambda)=\Psi^{T}(\lambda) e_{k}$. Then, using (19) and for $\alpha \in \mathbf{C}^{p}$ arbitrary

$$
\begin{aligned}
& \left\langle g_{k}(\lambda), \Psi^{T}(\lambda) T_{n}^{-1} \overline{\Psi(\omega)} \alpha\right\rangle \\
& \quad=\frac{1}{2 \pi} \int_{-\pi}^{\pi} \alpha^{\star} \Psi^{T}(\omega) T_{n}^{-1} \overline{\Psi(\lambda)} \Psi^{T}(\lambda) e_{k} \mathrm{~d} \lambda \\
& =\alpha^{\star} \Psi^{T}(\omega) T_{n}^{-1}\left[\frac{1}{2 \pi} \int_{-\pi}^{\pi} \overline{\Psi(\lambda)} \Psi^{T}(\lambda) \mathrm{d} \lambda\right] e_{k} \\
& =\alpha^{\star} \Psi^{T}(\omega) e_{k}=\alpha^{\star} g_{k}(\omega) \quad \forall k=1, \ldots, n .
\end{aligned}
$$

In what follows, we will mainly be concerned with functions $f(\omega):[-\pi, \pi] \rightarrow \mathbf{C}^{p}$ that arise as the restriction of $f(z): \mathbf{C} \rightarrow \mathbf{C}^{p}$ to the domain $z=e^{j \omega}, \omega \in[-\pi, \pi]$. As such, this paper will alternate between notation $f(\omega), f\left(e^{j \omega}\right)$, and $f(z)$ as convenient.

Furthermore, in the case that will be exclusively considered in this paper where all the $g_{k}(z)$ in (18) have real valued Laurent expansion co-efficients (corresponding to dynamic systems with real valued impulse responses), then $T_{n}$ defined by (18) has all real valued entries and, hence

$$
T_{n}=\frac{1}{2 \pi} \int_{-\pi}^{\pi} \Psi(\lambda) \Psi^{\star}(\lambda) \mathrm{d} \lambda
$$

is an alternate formulation of (19) that will sometimes prove useful.

The relevance of these reproducing kernel ideas to the problem of quantification of variance error for frequency function estimates will be seen to stem from the formulation (19) and (22) since, as will be shown, when the prediction errors are white, then the associated variance error in the frequency domain can be expressed (modulo a known scalar factor) as the quadratic form in (19) and (22) for some particular choice of elements of $\Psi(z)$ which depends on the model structure.

Therefore, since the preceding lemma also establishes that the reproducing kernel, which is unique, can be expressed as (17), then this provides a means for exact quantification of variance error provided that an explicit expression for the orthonormal basis $\mathcal{B}_{k}(z)$ spanned by the elements of $\Psi(z)$ can be found. 
With this is in mind, in the scalar case $p=1$, the following lemma details an important situation in which an explicit formulation of the necessary orthonormal basis is available.

Lemma 3.2 (Orthonormal Basis for Fixed Denominator Spaces): Consider the space

$$
X_{n} \triangleq \operatorname{Span}\left\{\frac{z^{-1}}{L_{n}(z)}, \frac{z^{-2}}{L_{n}(z)}, \ldots, \frac{z^{-m}}{L_{n}(z)}\right\}
$$

where

$$
L_{n}(z)=\prod_{k=1}^{n}\left(1-\xi_{k} z^{-1}\right), \quad\left|\xi_{k}\right|<1
$$

for some set of specified poles $\left\{\xi_{1}, \ldots, \xi_{n}\right\}$ and where $m \geq n$. Then, it holds that

$$
X_{n}=\operatorname{Span}\left\{\mathcal{B}_{1}(z), \ldots, \mathcal{B}_{n}(z)\right\}
$$

where

$$
\begin{aligned}
\mathcal{B}_{k}(z) & \triangleq \frac{\sqrt{1-\left|\xi_{k}\right|^{2}}}{z-\xi_{k}} \cdot \phi_{k-1}(z), \quad k=1, \ldots, m \\
\phi_{k}(z) & \triangleq \prod_{\ell=1}^{k} \frac{1-\overline{\xi_{\ell}} z}{z-\xi_{\ell}} \quad \phi_{0}(z) \triangleq 1
\end{aligned}
$$

and with $\xi_{k}=0$ for $k=n+1, \ldots, m$. Furthermore, the functions $\left\{\mathcal{B}_{k}(z)\right\}$ defined in (26) satisfy $\left\langle\mathcal{B}_{k}, \mathcal{B}_{\ell}\right\rangle=\delta(k-\ell)$ and, hence, form an orthonormal basis for $X_{n}$.

Proof: See [19].

In this special but important situation when the space $X_{n}$ is defined by (23), there exists yet another characterization of the reproducing kernel which will be of great utility in later developments.

Lemma 3.3 (Christoffel-Darboux Formula [20]): The reproducing kernel for $X_{n}$ in (23) is given by

$$
\varphi_{n}(z, \mu)=\frac{1-\overline{\phi_{n}(\mu)} \phi_{n}(z)}{z \bar{\mu}-1} .
$$

Proof: See [21].

Finally, in the multidimensional situation, one example with $p=2$ will be especially important for later applications.

Lemma 3.4: Let $X_{n}$ be the subspace

$$
X_{n} \triangleq \operatorname{Span}=\left\{f_{1}, \ldots, f_{n}, g_{1}, \ldots, g_{m}\right\}
$$

where $f_{k}(z)=\left[\tilde{f}_{k}(z), 0\right]^{T}$ and $g_{k}(z)=\left[0, \tilde{g}_{k}(z)\right]^{T}$ with all the $\tilde{g}_{k}, \tilde{f}_{k}$ being scalar valued. Suppose that $\varphi_{n}^{g}(\lambda, \omega)$ and $\varphi_{m}^{f}(\lambda, \omega)$ are the reproducing kernels for

$$
X_{n}^{g} \triangleq \operatorname{Span}\left\{\tilde{g}_{1}, \ldots, \tilde{g}_{n}\right\} \quad X_{n}^{f} \triangleq \operatorname{Span}\left\{\tilde{f}_{1}, \ldots, \tilde{f}_{m}\right\}
$$

respectively. Then, the reproducing kernel for $X_{n}$ is

$$
\varphi_{n}(\lambda, \mu)=\left[\begin{array}{cc}
\varphi_{n}^{f}(\lambda, \omega) & 0 \\
0 & \varphi_{m}^{g}(\lambda, \omega)
\end{array}\right]
$$

Proof: Follows by inspection.

\section{B. Functions Associated With the Reproducing Kernel}

In this section, we will only be concerned with the scalar case $p=1$, in which case a natural generalization of the reproducing kernel $\varphi_{n}(\lambda, \omega)$ expressed as the quadratic form (22) is the associated quadratic form

$$
\Psi^{\star}\left(e^{j \omega}\right) T_{n}^{-1} Q_{n} T_{n}^{-1} \Psi\left(e^{j \lambda}\right)
$$

where

$$
Q_{n}=\frac{1}{2 \pi} \int_{-\pi}^{\pi} \Psi\left(e^{j \lambda}\right) \Psi^{\star}\left(e^{j \lambda}\right)\left|\tilde{H}\left(e^{j \lambda}\right)\right|^{2} d \lambda
$$

for some function $\tilde{H}$. As the next lemma shows, the quantity (31) can also be expressed in terms of the reproducing kernel $\varphi_{n}(\lambda, \omega)$. In the sequel, the utility of (32) and the following quantification will arise when considering variance errors with fixed and incorrect noise models.

Lemma 3.5: Consider the case of $\Psi(z)$ defined by (18) with $p=1$ (all the $\left\{g_{k}\right\}$ scalar valued) and with $\varphi_{n}(\lambda, \omega)$ being the reproducing kernel for the space $X_{n}$ defined in (12). Then, under the assumptions of Lemma 3.1 and for any $\tilde{H} \in L_{2}$ with associated matrix $Q_{n}$ defined in (32) it holds that

$$
\Psi^{\star}\left(e^{j \omega}\right) T_{n}^{-1} Q_{n} T_{n}^{-1} \Psi\left(e^{j \omega}\right)=\left\|\tilde{H}\left(e^{j \lambda}\right) \varphi_{n}(\lambda, \omega)\right\|^{2}
$$

with the implied integration in the norm being with respect to $\lambda$ while $\omega$ is fixed.

Proof: From Lemma 3.1, we have that the reproducing kernel for the space spanned by the elements of $\Psi(z)$ is given by

$$
\varphi_{n}(\lambda, \omega)=\Psi^{\star}\left(e^{j \omega}\right) T_{n}^{-1} \Psi\left(e^{j \lambda}\right) .
$$

Hence

$$
\begin{aligned}
\left\|\tilde{H}\left(e^{j \lambda}\right) \varphi_{n}(\lambda, \omega)\right\|^{2} \\
=\frac{1}{2 \pi} \int_{-\pi}^{\pi} \Psi^{\star}\left(e^{j \omega}\right) T_{n}^{-1} \Psi\left(e^{j \lambda}\right) \\
\quad \times \Psi^{\star}\left(e^{j \lambda}\right) T_{n}^{-1} \Psi\left(e^{j \omega}\right)\left|\tilde{H}\left(e^{j \lambda}\right)\right|^{2} d \lambda \\
=\Psi^{\star}\left(e^{j \omega}\right) T_{n}^{-1}\left[\frac{1}{2 \pi} \int_{-\pi}^{\pi} \Psi\left(e^{j \lambda}\right)\right. \\
\left.\quad \times \Psi^{\star}\left(e^{j \lambda}\right)\left|\tilde{H}\left(e^{j \lambda}\right)\right|^{2} \mathrm{~d} \lambda\right] T_{n}^{-1} \Psi\left(e^{j \omega}\right) \\
=\Psi^{\star}\left(e^{j \omega}\right) T_{n}^{-1} Q_{n} T_{n}^{-1} \Psi\left(e^{j \omega}\right) .
\end{aligned}
$$

Furthermore as the following lemma establishes, when the functions $\left\{g_{n}(z)\right\}$ defining the underlying space $X_{n}$ via (12) are of the form given in (24) for which Lemma 3.2 provides an orthonormalized form, then in this case it is possible to give a more explicit expression for the norm $\left\|\tilde{H}\left(e^{j \lambda}\right) \varphi_{n}(\lambda, \omega)\right\|$ that quantifies the quadratic form (31).

Lemma 3.6: Let $\varphi_{n}(\lambda, \omega)$ be the reproducing kernel for the space $X_{n}$ defined in (23). Then, for any function $\tilde{H} \in L_{2}$ with all poles strictly inside a disk $\{z \in \mathbf{C}:|z|<1-\delta\}$ for some $\delta>0$

$$
\begin{aligned}
\left\|\tilde{H}\left(e^{j \lambda}\right) \varphi_{n}(\lambda, \omega)\right\|^{2}= & \left|\tilde{H}\left(e^{j \omega}\right)\right|^{2} \varphi_{n}(\omega, \omega) \\
& +2 \operatorname{Re}\left\{e^{j \omega} \gamma^{\prime}\left(e^{j \omega}\right)\right\}+R_{n}\left(e^{j \omega}\right)
\end{aligned}
$$


where $\gamma(z)$ denotes the positive real part of $\tilde{H}(z) \tilde{H}\left(z^{-1}\right)$ which is the unique function that is analytic outside the unit disk such that

$$
\tilde{H}(z) \tilde{H}\left(z^{-1}\right)=\gamma(z)+\gamma\left(z^{-1}\right) .
$$

Furthermore

$$
\begin{aligned}
& R_{n}\left(e^{j \omega}\right)=2 \operatorname{Re}\left\{e^{j \omega} \phi_{n}\left(e^{j \omega}\right) \frac{1}{2 \pi j}\right. \\
&\left.\times \oint_{|z|=1-\delta} \frac{\overline{\phi_{n}(z)}}{\left(z-e^{j \omega}\right)^{2}} \gamma(z) d z\right\} .
\end{aligned}
$$

Here, $\phi_{n}(z)$ is the Blaschke product defined in (26) and $\delta>0$ is chosen such that the region $|z| \geq 1-\delta$ does not include any of the poles $\left\{\xi_{k}\right\}$ or any of the poles of $\tilde{H}(z)$.

Proof: See Appendix A.

In several important applications, it is important to realize that by virtue of the term $R_{n}\left(e^{j \omega}\right)$ being zero, the quantification (35) can be simplified, as made explicit in the following final result of this section.

Corollary 3.1: The function $R_{n}\left(e^{j \omega}\right)$ in (35) vanishes under the restriction that with $\alpha(z), \beta(z)$ being polynomials in $z^{-1}$, then $\widetilde{H}(z)=\beta(z) \alpha^{-1}(z)$ with all the zeros of $\alpha(z)$ taken (without replacement) from the set $\left\{\xi_{1}, \ldots, \xi_{n}\right\}$ defining the space $X_{n}$ via (23), (24), and the order of $\beta(z)$ being no greater than $\alpha(z)$.

Proof: Via (36), any pole of $\gamma(z)$ is also a pole of $\tilde{H}(z)$, which by the assumptions of the lemma are contained within the zeros of $\overline{\phi_{n}(z)}$. Therefore, in this case the integrand in (37) is analytic within the contour of integration and, hence, the integral and, hence, $R_{n}\left(e^{j \omega}\right)$ is zero.

\section{Problem Formulation}

With the necessary technical tools now established, the paper proceeds to precisely define the format of the estimation problem considered here and to provide the links between it and the preceding material.

In what follows, it is assumed that the relationship between an observed input data record $\left\{u_{t}\right\}$ and output data record $\left\{y_{t}\right\}$ is modeled according to

$$
\mathcal{M}: y_{t}=G\left(q, \theta^{n}\right) u_{t}+H\left(q, \theta^{n}\right) e_{t}
$$

where the "dynamics model" $G\left(q, \theta^{n}\right)$ and the "noise model" $H\left(q, \theta^{n}\right)$ are jointly parametrized by a vector $\theta^{n} \in \mathbf{R}^{n}$ and are of the rational forms $\left(A\left(q, \theta^{n}\right)-D\left(q, \theta^{n}\right)\right.$ are all polynomials in the backward shift operator $q^{-1}$ )

$$
G\left(q, \theta^{n}\right)=\frac{B\left(q, \theta^{n}\right)}{A\left(q, \theta^{n}\right)} \quad H\left(q, \theta^{n}\right)=\frac{C\left(q, \theta^{n}\right)}{D\left(q, \theta^{n}\right)}
$$

while $\left\{e_{t}\right\}$ in (38) is a zero-mean white noise sequence that satisfies $\mathrm{E}\left\{e_{t}^{2}\right\}=\sigma^{2}, \mathrm{E}\left\{\left|e_{t}\right|^{8}\right\}<\infty$.

The postulated relationship (38) can encompass a range of model structures such as FIR, ARMAX, "output-error," and "Box-Jenkins" [2], [22], [3]. For all these cases, since $H\left(q, \theta^{n}\right)$ is also constrained to be monic (i.e., $\lim _{|q| \rightarrow \infty} H\left(q, \theta^{n}\right)=1$ ), then the mean-square optimal one-step ahead predictor $\hat{y}_{t}\left(\theta^{n}\right)$ based on the model structure (38) is [2]

$$
\hat{y}_{t}\left(\theta^{n}\right)=H^{-1}\left(q, \theta^{n}\right) G\left(q, \theta^{n}\right) u_{t}+\left[1-H^{-1}\left(q, \theta^{n}\right)\right] y_{t}
$$

with associated prediction error

$$
\varepsilon_{t}\left(\theta^{n}\right) \triangleq y_{t}-\hat{y}_{t}\left(\theta^{n}\right)=H^{-1}\left(q, \theta^{n}\right)\left[y_{t}-G\left(q, \theta_{n}\right) u_{t}\right] .
$$

Using this, a quadratic estimation criterion may be defined as

$$
V_{N}\left(\theta^{n}\right)=\frac{1}{2 N} \sum_{t=1}^{N} \varepsilon_{t}^{2}\left(\theta^{n}\right)
$$

and then used to construct the prediction error estimate $\hat{\theta}_{N}^{n}$ of $\theta^{n}$ as

$$
\hat{\theta}_{N}^{n} \triangleq \underset{\theta^{n} \in \Theta}{\arg \min } V_{N}\left(\theta^{n}\right)
$$

where $\Theta \subset \mathbf{R}^{n}$ is compact. Forming system estimates via the formulation (38)-(43) has become quite standard, in large part due to the availability of sophisticated software tools implementing the method [23], but also due to considerations such as statistical efficiency, and further extensive understanding of the theoretical properties of such an approach [24], [2], [22].

For example, as has been established in [25] and [2], under certain mild assumptions on the nature of the input $\left\{u_{t}\right\}$, the estimate $\hat{\theta}_{N}^{n}$ converges with increasing $N$ according to

$$
\lim _{N \rightarrow \infty} \hat{\theta}_{N}^{n}=\theta_{\circ}^{n} \triangleq \underset{\theta^{n} \in \Theta}{\arg \min } \lim _{N \rightarrow \infty} \mathrm{E}\left\{V_{N}\left(\theta^{n}\right)\right\} \quad \text { w.p.1. }
$$

and in what follows, it will be assumed that $\theta_{\circ}^{n}$ defined by (44) is unique. As well, it also holds that as $N$ increases, the estimate $\hat{\theta}_{N}^{n}$ converges in law to a Normally distributed random variable with mean value $\theta_{\circ}^{n}$ according to [26], [22], [2]

$$
\sqrt{N}\left(\hat{\theta}_{N}^{n}-\theta_{\circ}^{n}\right) \stackrel{\mathcal{D}}{\rightarrow} \mathcal{N}\left(0, P_{n}\right) \quad \text { as } N \rightarrow \infty
$$

and, furthermore, under the added assumption of $\mathrm{E}\left\{\left|e_{t}\right|^{8}\right\}<\infty$ then as established in [2, App. 9B]

$$
\lim _{N \rightarrow \infty} \operatorname{Var}\left\{\hat{\theta}_{N}^{n}-\theta_{\circ}^{n}\right\}=P_{n} .
$$

The matrix $P_{n}$, which gives a measure of parameter space estimation error, is of central importance to this paper. Its formulation is, in general, problem specific, but in the particular case of the model structure (38) being rich enough to encompass any true underlying dynamics [2]

$$
P_{n}^{-1}=\frac{1}{\sigma^{2}} \mathrm{E}\left\{\psi_{t}\left(\theta_{\circ}^{n}\right) \psi_{t}^{T}\left(\theta_{\circ}^{n}\right)\right\}
$$

where for some matrix of transfer functions $\Pi\left(q, \theta^{n}\right)$, and some quasistationary (possibly vector valued) signal $\zeta_{t}\left(\theta^{n}\right)$

$$
\begin{aligned}
\psi_{t}\left(\theta^{n}\right) & \triangleq-\frac{\mathrm{d}}{\mathrm{d} \theta^{n}} \hat{y}_{t}\left(\theta^{n}\right) \\
& =-H^{-1}\left(q, \theta^{n}\right) \frac{\mathrm{d} \Pi\left(q, \theta^{n}\right)}{\mathrm{d} \theta^{n}} \zeta_{t}\left(\theta^{n}\right) .
\end{aligned}
$$

Unfortunately, while this explicit formulation of $P_{n}$ exists, in general it does not provide significant insight into how various design variables affect the accuracy of the estimated frequency functions $G\left(e^{j \omega}, \hat{\theta}_{N}^{n}\right)$ and $H\left(e^{j \omega}, \hat{\theta}_{N}^{n}\right)$. In response to this, the 
seminal work [27], [4], [5], [2], [6] has used an approach of investigating how (45) manifests itself in the variability $\Delta_{n}(\omega)$ of $G\left(e^{j \omega}, \hat{\theta}_{N}^{n}\right)$ and $H\left(e^{j \omega}, \hat{\theta}_{N}^{n}\right)$; the result being approximations such as (2).

Central to the contribution of this paper is the novel approach of recognising that the problem of quantifying $\Delta_{n}(\omega)$ is closely related to the problem of quantifying the reproducing kernel for a certain space $X_{n}$ which is defined via the rows of the matrix ( $\theta^{n}$ is assumed to be a column vector)

$$
\left.\Psi\left(z, \theta_{\circ}^{n}\right) \triangleq H^{-1}\left(z, \theta_{\circ}^{n}\right) \frac{\mathrm{d} \Pi\left(z, \theta^{n}\right)}{\mathrm{d} \theta^{n}}\right|_{\theta^{n}=\theta_{\circ}^{n}} S_{\zeta_{\circ}}(z)
$$

according to

$$
X_{n} \triangleq \operatorname{Span}\left\{\left[\Psi\left(z, \theta_{\circ}^{n}\right)\right]_{1}^{T}, \ldots,\left[\Psi\left(z, \theta_{\circ}^{n}\right)\right]_{n}^{T}\right\}
$$

and where, in (49), the term $S_{\zeta_{\circ}}(z)$ is a spectral factor (under mild assumptions, this factor will be unique) associated with the process $\left\{\zeta_{t}\left(\theta_{\circ}^{n}\right)\right\}$.

To make this more concrete, and for future use in the following sections, the space $X_{n}$ for certain important model structures is provided in the following lemmas.

Lemma 4.1 (Characterization of Space: Box-Jenkins Structure): Suppose that the model structure (38) is parametrized with polynomials of the form

$$
\begin{aligned}
& A\left(q, \theta^{n}\right)=1+a_{1} q^{-1}+a_{2} q^{-1}+\cdots+a_{m_{a}} q^{-m_{a}} \\
& B\left(q, \theta^{n}\right)=b_{1} q^{-1}+b_{2} q^{-1}+\cdots+b_{m_{b}} q^{-m_{b}} \\
& D\left(q, \theta^{n}\right)=1+d_{1} q^{-1}+d_{2} q^{-1}+\cdots+d_{m_{d}} q^{-m_{d}} \\
& C\left(q, \theta^{n}\right)=1+c_{1} q^{-1}+c_{2} q^{-1}+\cdots+c_{m_{c}} q^{-m_{c}}
\end{aligned}
$$

for some integers $m_{a}, m_{b}, m_{c}, m_{d}$. Then, (48) holds with

$$
\Pi\left(q, \theta^{n}\right)=\left[G\left(q, \theta^{n}\right), H\left(q, \theta^{n}\right)\right] \quad \zeta_{t}\left(\theta^{n}\right)=\left[\begin{array}{c}
u_{t} \\
\varepsilon_{t}\left(\theta^{n}\right)
\end{array}\right]
$$

and, therefore, with $F(z)$ being a spectral factor of the input spectrum of $\Phi_{u}(\omega)$, and in the case where $\Phi_{u e} \equiv 0$, the space $X_{n}$ defined in (50), (49) may be expressed as

$$
\begin{array}{r}
X_{n}=\operatorname{Span}\left\{f_{1}(z), \ldots, f_{m_{a}+m_{b}}(z),\right. \\
\left.g_{1}(z), \ldots, g_{m_{c}+m_{d}}(z)\right\}
\end{array}
$$

where

$$
\begin{aligned}
& f_{k}(z) \triangleq\left[\frac{z^{-k} F(z)}{A^{2}\left(z, \theta_{\circ}^{n}\right) H\left(z, \theta_{\circ}^{n}\right)}, 0\right]^{T} \\
& g_{k}(z) \triangleq\left[0, \frac{z^{-k}}{C\left(z, \theta_{\circ}^{n}\right) D\left(z, \theta_{\circ}^{n}\right)}\right]^{T} .
\end{aligned}
$$

Proof: See Appendix B.1.

Lemma 4.2 (Characterization of Space: Output-Error Structure): Suppose that the model structure (38) is parametrized with the numerator and denominator polynomials of the form (51), (52), and

$$
C\left(q, \theta^{n}\right)=D\left(q, \theta^{n}\right)=1
$$

for some integers $m_{a}, m_{b}$. Then, (48) holds with

$$
H\left(q, \theta_{\circ}^{n}\right)=1 \quad \Pi\left(q, \theta^{n}\right)=G\left(q, \theta^{n}\right) \quad \zeta_{t}\left(\theta^{n}\right)=u_{t}
$$

and, therefore, with $F(z)$ being a spectral factor of the input spectrum of $\Phi_{u}(\omega)$, the space $X_{n}$ defined in (50), (49) may be expressed as

$$
\begin{aligned}
X_{n}=\operatorname{Span}\left\{\frac{z^{-1} F(z)}{A^{2}\left(z, \theta_{\circ}^{n}\right)}, \frac{z^{-2} F(z)}{A^{2}\left(z, \theta_{\circ}^{n}\right)}, \ldots,\right. \\
\left.\frac{z^{-\left(m_{a}+m_{b}\right)} F(z)}{A^{2}\left(z, \theta_{\circ}^{n}\right)}\right\} .
\end{aligned}
$$

Proof: See Appendix B.2.

Lemma 4.3 (Characterization of Space-ARMAX Structure): Suppose that the model structure (38) is parametrized with the numerator and denominator polynomials of the form (51), (52), and

$$
\begin{aligned}
& D\left(q, \theta^{n}\right)=A\left(q, \theta^{n}\right), \\
& C\left(q, \theta^{n}\right)=1+c_{1} q^{-1}+c_{2} q^{-1}+\cdots+c_{m_{c}} q^{-m_{c}}
\end{aligned}
$$

for some integer $m_{c}$. Then, (48) holds with

$$
\begin{aligned}
\Pi\left(q, \theta^{n}\right) & =\left[\frac{\mathrm{d} G\left(q, \theta^{n}\right)}{\mathrm{d} \theta^{n}}, \frac{\mathrm{d} H\left(q, \theta^{n}\right)}{\mathrm{d} \theta^{n}}\right] \\
\zeta_{t}\left(\theta^{n}\right) & =\left[\begin{array}{c}
u_{t} \\
\varepsilon_{t}\left(\theta^{n}\right)
\end{array}\right]
\end{aligned}
$$

and, therefore, with $F(z)$ being a spectral factor of the input spectrum of $\Phi_{u}(\omega)$, in the case where $\Phi_{u e} \equiv 0$ the space $X_{n}$ defined in (50), (49) may be expressed as

$$
\begin{aligned}
X_{n}=\operatorname{Span}\left\{f_{1}(z), \ldots, f_{m_{a}}(z), g_{1}(z), \ldots\right. \\
\left.g_{m_{b}}(z), h_{1}(z), \ldots, h_{m_{c}}(z)\right\}
\end{aligned}
$$

where

$$
\begin{aligned}
f_{k}(z) & \triangleq\left[\frac{G\left(z, \theta_{\circ}^{n}\right) F(z) z^{-k}}{C\left(z, \theta_{\circ}^{n}\right)}, \frac{z^{-k}}{A\left(z, \theta_{\circ}^{n}\right)}\right]^{T} \\
g_{k}(z) & \triangleq\left[\begin{array}{ll}
\frac{F(z) z^{-k}}{C\left(z, \theta_{\circ}^{n}\right)} & 0
\end{array}\right]^{T} \\
h_{k}(z) & \triangleq\left[0, \frac{z^{-k}}{C\left(z, \theta_{\circ}^{n}\right)}\right]^{T} .
\end{aligned}
$$

Proof: See Appendix B.3.

Note that the requirement in the Box-Jenkins and ARMAX cases that $\Phi_{u e} \equiv 0$ is satisfied whenever data is collected under open loop conditions. Note also that no requirement on $\Phi_{u e}$ is stated for the case of output-error structure and, hence, the results to follow for this case will hold under arbitrary closed loop operating conditions.

Finally, in what follows it will also be important to consider the relationship between the true underlying system $\mathcal{S}$ given as

$$
\mathcal{S}: y_{t}=G(q) u_{t}+\nu_{t} \quad \nu_{t}=H(q) e_{t}
$$

and the model structure $\mathcal{M}$ defined by (38). 
In particular, when it is assumed that $\mathcal{S} \in \mathcal{M}$, then under the assumptions to follow it will hold that $\varepsilon_{t}\left(\theta_{\circ}^{n}\right)=e_{t}$ and that $G\left(q, \theta_{\circ}^{n}\right)=G(q), H\left(q, \theta_{\circ}^{n}\right)=H(q)$, in which case the latter shorter notation will often be used together with

$$
\Phi_{\nu}(\omega)=\sigma^{2}\left|H\left(e^{j \omega}\right)\right|^{2}
$$

representing the power spectral density of the process $\left\{\nu_{t}\right\}$.

\section{MAIN RESUltS}

With the necessary technical tools and problem formulation now established, the paper now proceeds to present the main results which are new quantifications such as (4) that are "exact" for finite-model order and permit analysis of the phenomenon illustrated in Fig. 1. In organizing this material, it will be convenient to discriminate whether the true system is in the model class or not.

\section{A. True System Contained in Model Structure $(\mathcal{S} \in \mathcal{M})$}

The central result to be employed here finally makes completely explicit the link between variance error and reproducing kernels, as hinted at in the previous sections. It applies for all cases encompassed by the model structure (38), including those of FIR, ARMAX, OE, and Box-Jenkins type.

Theorem 5.1 (Frequency Domain Variability-S $\in \mathcal{M}$ ): Suppose that $\hat{\theta}_{N}^{n}$ is calculated via (43) using the model structure (38) and that the following assumptions are satisfied.

1) $\varepsilon_{t}\left(\theta_{\circ}^{n}\right)=e_{t}$ where $\left\{e_{t}\right\}$ is a zero mean i.i.d. process that satisfies $\mathrm{E}\left\{\left|e_{t}\right|^{8}\right\}<\infty$.

2) The relationship (48) holds for some $\Pi\left(q, \theta^{n}\right)$, and some quasistationary (possibly vector valued) signal $\left\{\zeta_{t}\left(\theta^{n}\right)\right\}$ and for which the power spectral density $\Phi_{\zeta_{0}}(\omega)$ of $\left\{\zeta_{t}\left(\theta_{\circ}^{n}\right)\right\}$ satisfies $\Phi_{\zeta_{0}}(\omega)>0 \forall \omega \in[-\pi, \pi]$.

3) Neither of $G\left(z, \theta_{\circ}^{n}\right)$ or $H\left(z, \theta_{\circ}^{n}\right)$ contain any pole-zero cancellations.

Then denoting $S_{\zeta_{0}}(z)$ as a spectral factor of the power spectral density $\Phi_{\zeta_{\circ}}(\omega)$ of $\left\{\zeta_{t}\left(\theta_{\circ}^{n}\right)\right\}$

$$
\lim _{N \rightarrow \infty} N \cdot \operatorname{Cov}\left\{\left[\begin{array}{c}
G\left(e^{j \omega}, \hat{\theta}_{N}^{n}\right) \\
H\left(e^{j \omega}, \hat{\theta}_{N}^{n}\right)
\end{array}\right]\right\}=\Delta_{n}(\omega)
$$

where

$$
\Delta_{n}(\omega)=\Phi_{\nu}(\omega) S_{\zeta_{\circ}}^{-\star}\left(e^{j \omega}\right) \varphi_{n}(\omega, \omega) S_{\zeta_{\circ}}^{-1}\left(e^{j \omega}\right)
$$

with $\varphi_{n}(\lambda, \omega)$ being the reproducing kernel for the space $X_{n}$ defined via (49) and (50).

Proof: See Appendix C.

The problem of deriving an expression $\Delta_{n}(\omega)$ for the estimate covariance in the frequency domain is therefore established as being equivalent to the problem of quantifying the reproducing kernel for a certain space $X_{n}$ which is that spanned by the rows of $\Psi\left(z, \theta_{\circ}^{n}\right)$ defined in (49).

According to Lemmas 4.1-4.3 this space and, hence, the reproducing kernel $\varphi_{m}(\lambda, \omega)$, depends on the model structure employed. Therefore the covariance $\Delta_{n}(\omega)$ of the dynamic system estimate, will also depend on the model structure as will now be made explicit via the following corollaries to this main theorem.
Corollary 5.1 (Variability of Box-Jenkins Model $\mathcal{S} \in \mathcal{M}$ ): Suppose that all the conditions of Lemma 4.1 are satisfied and, hence, that a Box-Jenkins model structure is employed. Suppose further that the conditions of Theorem 5.1 are satisfied and that

$$
A_{\dagger}(z)=A^{2}\left(z, \theta_{\circ}^{n}\right) \frac{H\left(z, \theta_{\circ}^{n}\right)}{F(z)}
$$

is a polynomial in $z^{-1}$ of degree at most $m_{a}+m_{b}$. Define the zeros $\left\{\xi_{k}\right\}$ and $\left\{\eta_{k}\right\}$ according to

$$
\begin{aligned}
z^{m_{a}+m_{b}} A_{\dagger}(z) & =\prod_{k=1}^{m_{a}+m_{b}}\left(z-\xi_{k}\right) \\
z^{m_{c}+m_{d}} C\left(z, \theta_{\circ}^{n}\right) D\left(z, \theta_{\circ}^{n}\right) & =\prod_{k=1}^{m_{c}+m_{d}}\left(z-\eta_{k}\right)
\end{aligned}
$$

and use these to define the functions $\kappa(\omega)$ and $\widetilde{\kappa}(\omega)$ according to

$$
\begin{aligned}
& \kappa(\omega) \triangleq \sum_{k=1}^{m_{a}+m_{b}} \frac{1-\left|\xi_{k}\right|^{2}}{\left|e^{j \omega}-\xi_{k}\right|^{2}} \\
& \tilde{\kappa}(\omega) \triangleq \sum_{k=1}^{m_{c}+m_{d}} \frac{1-\left|\eta_{k}\right|^{2}}{\left|e^{j \omega}-\eta_{k}\right|^{2}} .
\end{aligned}
$$

Then

$$
\begin{aligned}
\lim _{N \rightarrow \infty} N \operatorname{Cov}\left\{\left[\begin{array}{l}
G\left(e^{j \omega}, \hat{\theta}_{N}^{n}\right) \\
H\left(e^{j \omega}, \hat{\theta}_{N}^{n}\right)
\end{array}\right]\right\} & \\
& =\Phi_{\nu}(\omega)\left[\begin{array}{cc}
\frac{\kappa(\omega)}{\Phi_{u}(\omega)} & 0 \\
0 & \frac{\widetilde{\kappa}(\omega)}{\sigma^{2}}
\end{array}\right] .
\end{aligned}
$$

Furthermore, regardless of whether (70) is satisfied for some polynomial $A_{\dagger}(z)$

$$
\lim _{N \rightarrow \infty} N \operatorname{Var}\left\{H\left(e^{j \omega}, \hat{\theta}_{N}^{n}\right)\right\}=\tilde{\kappa}(\omega)\left|H\left(e^{j \omega}\right)\right|^{2} .
$$

Proof: Since the conditions of Theorem 5.1 are satisfied, then the asymptotic in $N$ variance $\Delta_{n}(\omega)$ is given by (69). Furthermore, since the assumptions of Lemma 4.1 are also satisfied, then the quantity $\varphi_{n}(\lambda, \omega)$ in (69) is the reproducing kernel for the space $X_{n}$ defined in (56) and (57). However, since assumption (70) holds, then this space can be reformulated as

$$
\begin{aligned}
X_{n}= & \operatorname{Span}\left\{f_{1}(z), \ldots, f_{m_{a}+m_{b}}(z),\right. \\
& \left.g_{1}(z), \ldots, g_{m_{c}+m_{d}}(z)\right\} \\
f_{k}(z) \triangleq & {\left[\frac{z^{-k}}{A_{\dagger}(z)}, 0\right]^{T} } \\
g_{k}(z) \triangleq & {\left[0, \frac{z^{-k}}{C\left(z, \theta_{\circ}^{n}\right) D\left(z, \theta_{\circ}^{n}\right)}\right]^{T} . }
\end{aligned}
$$

Therefore, by Lemma 3.4 , the multivariable reproducing kernel for $X_{n}$ is of the form (30). In this case, (17) may be used to quantify the associated scalar reproducing kernels $\varphi_{n}^{f}(\lambda, \omega), \varphi_{n}^{g}(\lambda, \omega)$ via the formulation (26), and then setting $\kappa(\omega)=\varphi_{n}^{f}(\omega, \omega), \tilde{\kappa}(\omega)=\varphi_{n}^{g}(\omega, \omega)$ completes the proof. 
Corollary 5.2 (Variability of Output-Error Model $\mathcal{S} \in \mathcal{M}$ ): Suppose that all the conditions of Lemma 4.2 are satisfied and, hence, that an output-error model structure is employed. Suppose further that the conditions of Theorem 5.1 are satisfied and that

$$
A_{\dagger}(z)=\frac{A^{2}\left(z, \theta_{\circ}^{n}\right)}{F(z)}
$$

is a polynomial in $z^{-1}$ of degree at most $m_{a}+m_{b}$. Define the zeros $\left\{\xi_{k}\right\}$ and the function $\kappa(\omega)$ according to

$$
\begin{aligned}
z^{m_{a}+m_{b}} A_{\dagger}(z) & =\prod_{k=1}^{m_{a}+m_{b}}\left(z-\xi_{k}\right) \\
\kappa(\omega) & \triangleq \sum_{k=1}^{m_{a}+m_{b}} \frac{1-\left|\xi_{k}\right|^{2}}{\left|e^{j \omega}-\xi_{k}\right|^{2}} .
\end{aligned}
$$

Then

$$
\lim _{N \rightarrow \infty} N \cdot \operatorname{Var}\left\{G\left(e^{j \omega}, \hat{\theta}_{N}^{n}\right)\right\}=\sigma^{2} \cdot \frac{\kappa(\omega)}{\Phi_{u}(\omega)} .
$$

Proof: Since the conditions of Theorem 5.1 are satisfied, then the asymptotic in $N$ variance $\Delta_{n}(\omega)$ is given by (69). Furthermore, since the assumptions of Lemma 4.2 are also satisfied, then the quantity $\varphi_{n}(\lambda, \omega)$ in (69) is the reproducing kernel for the space $X_{n}$ defined in (60). However, since assumption (70) holds, then this space can be reformulated as

$$
X_{n}=\operatorname{Span}\left\{\frac{z^{-k}}{A_{\dagger}(z)}, \ldots, \frac{z^{-\left(m_{a}+m_{b}\right)}}{A_{\dagger}(z)}\right\} .
$$

Therefore, by Lemma 3.1, the reproducing kernel $\varphi_{n}(\lambda, \omega)$ for $X_{n}$ is of the form (17) with the associated orthonormal basis $\left\{\mathcal{B}_{k}(z)\right\}$ given by Lemma 3.2. Setting $\kappa(\omega)=\varphi_{n}(\omega, \omega)$ then completes the proof.

The essential implication of these corollaries are that although the equality in the variance expressions (73), (79) depends on $N$ being infinitely large, it could be expected that for finite $N$ the left- and right-hand sides of (73), (79) should be approximately equal with the limiting operations removed, so that the following quantifications are useful:

$$
\begin{aligned}
& \mathrm{E}\left\{\left|G\left(e^{j \omega}, \hat{\theta}_{N}^{n}\right)-G\left(e^{j \omega}\right)\right|^{2}\right\} \\
& \approx \frac{1}{N} \frac{\hat{\sigma}^{2}\left|H\left(e^{j \omega}, \hat{\theta}_{N}^{n}\right)\right|^{2}}{\Phi_{u}(\omega)} \sum_{k=1}^{m_{a}+m_{b}} \frac{1-\left|\xi_{k}\right|^{2}}{\left|e^{j \omega}-\xi_{k}\right|^{2}} \\
& \mathrm{E}\left\{\left|H\left(e^{j \omega}, \hat{\theta}_{N}^{n}\right)-H\left(e^{j \omega}\right)\right|^{2}\right\} \\
& \approx \frac{1}{N}\left|H\left(e^{j \omega}, \hat{\theta}_{N}^{n}\right)\right|^{2} \sum_{k=1}^{m_{c}+m_{d}} \frac{1-\left|\eta_{k}\right|^{2}}{\left|e^{j \omega}-\eta_{k}\right|^{2}}
\end{aligned}
$$

where

$$
\hat{\sigma}^{2} \triangleq \frac{1}{N} \sum_{t=1}^{N} \varepsilon_{t}^{2}\left(\hat{\theta}_{N}^{n}\right) .
$$

There are some important facets of, and conclusions to be drawn from these quantifications.

- First, and most importantly, the expressions (81) and (82) are "exact"for finite-model order in the sense that, unlike most pre-existing results such as (2), they are not derived from an asymptotic in model order argument. As such, they are likely to be far more accurate for practical cases of finite, and indeed low model order as has already been illustrated in Fig. 1.

- Second, these results in Theorem 5.1 and Corollary 5.1 represent an extension of those given in [10]. There, model structures with poles fixed at those of the true underlying system, and fixed noise model were considered. Here, we consider the more general case where the poles and the noise model are unknown and, hence, estimated.

- Third, note that a key point is that the quantification (81) resolves an outstanding paradox in the theory of system identification. Namely, a consequence of the existing quantification (2) is that, since it applies for any shift invariant structure which includes the FIR and Box-Jenkins cases, then (as explained in [10]) it suggests that there is no variance penalty to be paid for estimating pole locations in the Box-Jenkins model structure (38), as opposed to fixing those pole locations and estimating just a numerator term via an FIR structure.

This is counterintuitive, and indeed the expression (81) indicates that it is in fact untrue. Specifically, consider the case of white input and white noise, i.e., $\Phi_{u}(\omega)=$ $\Phi_{\nu}(\omega) \equiv 1$. Then, in the case of the denominator order $m_{a}$ equalling the numerator order $m_{b}$, the results of [10] applying for the case of fixed denominator modeling with poles at $\left\{\xi_{k}\right\}$ (as well as Theorem 5.1) give an exact quantification for finite numerator order $m=m_{b}=m_{a}$ of

$$
\begin{aligned}
\lim _{N \rightarrow \infty} N \mathrm{E}\left\{\mid G\left(e^{j \omega}, \hat{\theta}_{N}^{n}\right)-\right. & \left.\left.G\left(e^{j \omega}\right)\right|^{2}\right\} \\
& =\frac{\sigma^{2}}{\Phi_{u}(\omega)} \sum_{k=1}^{m} \frac{1-\left|\xi_{k}\right|^{2}}{\left|e^{j \omega}-\xi_{k}\right|^{2}}
\end{aligned}
$$

whereas when the denominator is estimated, $A^{2}\left(z, \theta_{\circ}^{n}\right) H\left(z, \theta_{\circ}^{n}\right) / F(z)=A^{2}\left(z, \theta_{\circ}^{n}\right)$ and Corollary 5.1 then asserts that

$$
\begin{gathered}
\lim _{N \rightarrow \infty} N E\left\{\left|G\left(e^{j \omega}, \hat{\theta}_{N}^{n}\right)-G\left(e^{j \omega}\right)\right|^{2}\right\} \\
=\frac{\sigma^{2}}{\Phi_{u}(\omega)} \sum_{k=1}^{2 m} \frac{1-\left|\xi_{k}\right|^{2}}{\left|e^{j \omega}-\xi_{k}\right|^{2}} \\
=2 \cdot \frac{\sigma^{2}}{\Phi_{u}(\omega)} \sum_{k=0}^{m} \frac{1-\left|\xi_{k}\right|^{2}}{\left|e^{j \omega}-\xi_{k}\right|^{2}} .
\end{gathered}
$$

Therefore, via (84) and (85), the new quantification (81) of this paper is an expression that, as well as being exact for finite model orders $m_{a}, m_{b}$, is exactly double that pertaining to the case examined in [10] were poles are fixed. Since, roughly speaking, twice as much information (i.e., 
a numerator and a denominator) is being estimated, this new result now corroborates intuitive belief and resolves the aforementioned paradox.

Finally, note that if the underlying system is, in fact, of FIR type so that all the poles $\left\{\xi_{k}\right\}$ are at the origin, then (85) implies

$$
\mathrm{E}\left\{\left|G\left(e^{j \omega}, \hat{\theta}_{N}^{n}\right)-G\left(e^{j \omega}\right)\right|^{2}\right\} \approx 2 \cdot \frac{m \sigma^{2}}{N \Phi_{u}(\omega)}
$$

Therefore, modulo a factor of 2, there is a rapprochement in the FIR modeling case between the new quantifications derived here, and the pre-existing one (2).

In relation to this last point where $\Phi_{u}$ was assumed white, there are a range of nontrivial possibilities of input spectrum for which exact variance quantification is possible, as illustrated by the further corollary to Theorem 5.1.

Corollary 5.3 (Box-Jenkins Model, Colored Input Spectrum, $\mathcal{S} \in \mathcal{M})$ : Suppose that all the conditions of Lemma 4.1 and Theorem 5.1 are satisfied, and that the spectral factor $F(z)$ of $\Phi_{u}(\omega)$ and the limiting noise model $H\left(z, \theta_{\circ}^{n}\right)$ are of the form $(\mu \in \mathbf{C})$

$$
\begin{aligned}
F(z) & =\frac{\mu}{L(z)} \quad L(z)=\prod_{k=1}^{m_{f}}\left(1-f_{k} z^{-1}\right) \\
H\left(z, \theta_{\circ}^{n}\right) & =\prod_{k=1}^{m_{h}}\left(1-h_{k} z^{-1}\right) .
\end{aligned}
$$

Then, if $m_{b}=m_{a}+m_{f}+m_{h}+m_{\star}, m_{\star} \geq 0$

$$
\begin{aligned}
\lim _{N \rightarrow \infty} N \cdot \operatorname{Cov}\left\{\left[\begin{array}{l}
G\left(e^{j \omega}, \hat{\theta}_{N}^{n}\right) \\
H\left(e^{j \omega}, \hat{\theta}_{N}^{n}\right)
\end{array}\right]\right\} & \\
= & \Phi_{\nu}(\omega)\left[\begin{array}{cc}
\frac{\rho(\omega)}{\Phi_{u}(\omega)} & 0 \\
0 & \frac{\tilde{\kappa}(\omega)}{\sigma^{2}}
\end{array}\right]
\end{aligned}
$$

where, with the $\left\{\xi_{k}\right\}$ being chosen as the zeros of $A\left(z, \theta_{\circ}^{n}\right)$

$$
\begin{aligned}
\rho(\omega) \triangleq m_{\star}+2 & \cdot \sum_{k=1}^{m_{a}} \frac{1-\left|\xi_{k}\right|^{2}}{\left|e^{j \omega}-\xi_{k}\right|^{2}} \\
& +\sum_{k=1}^{m_{f}} \frac{1-\left|f_{k}\right|^{2}}{\left|e^{j \omega}-f_{k}\right|^{2}}+\sum_{k=1}^{m_{h}} \frac{1-\left|h_{k}\right|^{2}}{\left|e^{j \omega}-h_{k}\right|^{2}}
\end{aligned}
$$

and $\tilde{\kappa}(\omega)$ is defined in (72) of Theorem 5.1.

Proof: Clearly $A_{\dagger}(z)=A^{2}\left(z, \theta_{\circ}^{n}\right) H\left(z, \theta_{\circ}^{n}\right) / F(z)$ is a polynomial in $z^{-1}$ of degree $2 m_{a}+m_{h}+m_{f}$ which is less than $m_{a}+m_{b}$ under the given assumptions. Therefore, the corollary is established as an application of Theorem 5.1 upon noticing that $z^{m_{a}+m_{b}} A_{\dagger}(z)$ has $m_{\star}$ zeros at the origin with the remainder being the same as the zeros of $A^{2}\left(z, \theta_{\circ}^{n}\right) H\left(z, \theta_{\circ}^{n}\right) L(z)$.

The preceding corollary shows that when $\mathcal{S} \in \mathcal{M}$ and the input is of $\operatorname{AR}(p)$ type with noise of $\mathrm{MA}(r)$ type, then provided that $p+r$ more "lags" are modeled in the numerator $B\left(q, \theta^{n}\right)$ than the denominator $A\left(q, \theta^{n}\right)$, then the quantification

$$
\begin{aligned}
& E\left\{\left|G\left(e^{j \omega}, \hat{\theta}_{N}^{n}\right)-G\left(e^{j \omega}\right)\right|^{2}\right\} \\
& \approx \frac{\sigma^{2}}{N} \frac{\left|H\left(e^{j \omega}, \hat{\theta}_{N}^{n}\right)\right|^{2}}{\Phi_{u}(\omega)}\left[m_{\star}+2 \cdot \sum_{k=1}^{m_{a}} \frac{1-\left|\xi_{k}\right|^{2}}{\left|e^{j \omega}-\xi_{k}\right|^{2}}\right. \\
&\left.+\sum_{k=1}^{p} \frac{1-\left|\ell_{k}\right|^{2}}{\left|e^{j \omega}-\ell_{k}\right|^{2}}+\sum_{k=1}^{r} \frac{1-\left|c_{k}\right|^{2}}{\left|e^{j \omega}-c_{k}\right|^{2}}\right]
\end{aligned}
$$

is "exact" for finite-model order. This is a direct extension of the results of [10] from the fixed-and-correct denominator and noise model case considered there, to the more general Box-Jenkins modeling situation in which the denominator and noise model are both estimated.

Furthermore, comparing (89) to the quantification presented in [10] establishes that the effect of modeling the denominator is to double the size of the component $\sum_{k=1}^{m_{a}}\left(1-\left|\xi_{k}\right|^{2}\right) /\left|e^{j \omega}-\xi_{k}\right|^{2}$ (which depends on the poles $\left\{\xi_{k}\right\}$ of $G\left(q, \theta_{\circ}^{n}\right)$ ) in the variance of the dynamics estimate, and as mentioned in the introduction and previous discussion, this makes intuitive sense.

An important special case of the model structure (38) is the so-called output-error structure in which $m_{c}=m_{d}=0$ so that $H\left(q, \theta^{n}\right)=1$. The exact in model order variance quantifications for this modeling choice are worth stating separately for the sake of clarity.

Corollary 5.4 (Output-Error, Colored Input Spectrum $\mathcal{S} \in$ $\mathcal{M})$ : Suppose that all the conditions of Lemma 4.2 and Theorem 5.1 are satisfied and, hence, that an Output Error model structure is employed. Suppose further that a spectral factor $F(z)$ of $\Phi(\omega)$ is of the $\operatorname{AR}\left(m_{f}\right)$ form given in (86). Then, if the model structure (38) is used with $m_{c}=m_{d}=0$ and $m_{b}=m_{a}+m_{f}+m_{\star}, m_{\star} \geq 0$

$$
\begin{aligned}
& \lim _{N \rightarrow \infty} N \cdot \operatorname{Var}\left\{G\left(e^{j \omega}, \hat{\theta}_{N}^{n}\right)\right\} \\
& \quad=\frac{\sigma^{2}}{\Phi_{u}(\omega)}\left[m_{\star}+2 \cdot \sum_{k=1}^{m_{a}} \frac{1-\left|\xi_{k}\right|^{2}}{\left|e^{j \omega}-\xi_{k}\right|^{2}}+\sum_{k=1}^{m_{f}} \frac{1-\left|f_{k}\right|^{2}}{\left|e^{j \omega}-f_{k}\right|^{2}}\right]
\end{aligned}
$$

where the $\left\{\xi_{k}\right\}$ are the zeros of $A\left(q, \theta_{\circ}^{n}\right)$.

Proof: For the output-error case, $H\left(z, \theta_{\circ}^{n}\right)=1$ and, hence, under the assumptions on $F(z)$

$$
A_{\dagger}(z)=A^{2}\left(z, \theta_{\circ}^{n}\right) \frac{1}{F(z)}
$$

is a polynomial of order $m_{a}+m_{b}-m_{\star}$ that satisfies the order condition in Theorem 5.1. Furthermore, since the assumptions of Lemma 4.2 are also satisfied, then the quantity $\varphi_{n}(\lambda, \omega)$ in (69) is the reproducing kernel for the space $X_{n}$ defined in (60). However, since (91) holds, then this space can be reformulated as

$$
X_{n}=\operatorname{Span}\left\{\frac{z^{-1}}{A_{\dagger}(z)}, \ldots, \frac{z^{-m_{a}+m_{b}}}{A_{\dagger}(z)}\right\}
$$


Furthermore, (17) may be used to quantify the associated reproducing kernel $\varphi_{n}(\lambda, \omega)$ via the formulation (26). Substituting this in (69) and observing that $z^{m_{a}+m_{b}} A_{\dagger}(z)$ has $m_{\star}$ zeros at the origin, with the remainder being the zeros of $A^{2}\left(z, \theta_{\circ}^{n}\right) / F(z)$ then completes the proof.

\section{B. True System Not in Model Class $\mathcal{S} \notin \mathcal{M}$}

There is an important further situation to be considered, in which the noise model is fixed at a possibly incorrect value and, hence, $\mathcal{S} \notin \mathcal{M}$. In this case, the results of Theorem 5.1 are no longer valid, but exact (for finite model order) expressions for the frequency domain variability $\Delta_{n}(\omega)$ can also be established for this extended situation according to the following theorem.

Theorem 5.2 (Frequency Domain Variability $\mathcal{S} \notin$ $\mathcal{M})$ : Suppose that $\hat{\theta}_{N}^{n}$ is calculated via (43) using the model structure (38) with the modification that the noise model is fixed at $H(q, \theta)=H_{\star}(q)$ which is not necessarily equal to any true underlying one. Suppose further that Assumption 2 of Theorem 5.1 holds together with the following conditions.

1) The observed data obeys a model $y_{t}=G(q) u_{t}+H(q) e_{t}$ for some stable and inversely stable monic transfer function $H(q)$ and some $G(q)=G\left(q, \theta_{0}^{n}\right)$ with $\left\{e_{t}\right\}$ being a zero mean i.i.d. process that satisfies $\mathrm{E}\left\{\left|e_{t}\right|^{8}\right\}<\infty$.

2) $e_{t}$ and $u_{t}$ are jointly quasistationary with cross-spectrum $\Phi_{u e} \equiv 0$.

3) $G\left(z, \theta_{\circ}^{n}\right)$ does not contain any pole-zero cancellations.

Then

$$
\begin{aligned}
\lim _{N \rightarrow \infty} N \cdot \operatorname{Cov}\left\{G\left(e^{j \omega}, \hat{\theta}_{N}^{n}\right)\right\} \\
=\sigma^{2} \frac{\left|H_{\star}\left(e^{j \omega}\right)\right|^{2}}{\Phi_{u}(\omega)}\left\|\frac{H\left(e^{j \lambda}\right)}{H_{\star}\left(e^{j \lambda}\right)} \varphi_{n}(\lambda, \omega)\right\|^{2}
\end{aligned}
$$

where $\varphi_{n}(\lambda, \omega)$ is the reproducing kernel for the space $X_{n}$ defined by (50) with the specification (49) for $\Psi\left(z, \theta_{\circ}^{n}\right)$ replaced by

$$
\Psi\left(z, \theta_{\circ}^{n}\right)=\left.H_{\star}^{-1}(z) \frac{\mathrm{d} G\left(q, \theta^{n}\right)}{\mathrm{d} \theta^{n}}\right|_{\theta^{n}=\theta_{\circ}^{n}} F(z) .
$$

Proof: See Appendix D.

This theorem then has two corollaries that quantify frequency domain variability for the case of fixed, possibly incorrect noise model. The first corollary provides a quantification (again, exact for finite-model order) that applies under certain assumptions on the relationship between the fixed noise model $H_{\star}(z)$ and the input spectral factor $F(z)$.

Corollary 5.5 (Frequency Domain Variability-Fixed Noise Model, $\mathcal{S} \notin \mathcal{M}$ ): Suppose that the assumptions of Theorem 5.2 hold. Suppose further that

$$
A_{\dagger}(z)=A^{2}\left(z, \theta_{\circ}^{n}\right) \frac{H_{\star}(z)}{F(z)}
$$

is a polynomial in $z^{-1}$ of degree at most $m_{a}+m_{b}$, which is then used to define the zeros $\left\{\xi_{k}\right\}$ according to (71), and the functions $\kappa(\omega)$ and $\phi_{n}(z)$ (with $n=m_{a}+m_{b}$ ) according to (72), (26). Then

$$
\begin{aligned}
& \lim _{N \rightarrow \infty} N \cdot \operatorname{Cov}\left\{G\left(e^{j \omega}, \hat{\theta}_{N}^{n}\right)\right\} \\
& \quad=\frac{\Phi_{\nu}(\omega)}{\Phi_{u}(\omega)} \cdot \kappa(\omega)+\frac{\sigma^{2}\left|H_{\star}\left(e^{j \omega}\right)\right|^{2}}{\Phi_{u}(\omega)} \times \\
& 2 \operatorname{Re}\left\{e^{j \omega} \gamma^{\prime}\left(e^{j \omega}\right)+\frac{1}{2 \pi j} \oint_{\Gamma} \frac{e^{j \omega} \overline{\phi_{n}(z)} \phi_{n}\left(e^{j \omega}\right)}{\left(z-e^{j \omega}\right)^{2}} \gamma(z) \mathrm{d} z\right\}
\end{aligned}
$$

where $\gamma(z)$ is the positive real part of $H(z) H\left(z^{-1}\right) H_{\star}^{-1}(z) H_{\star}^{-1}\left(z^{-1}\right)$ that is analytic on $|z| \geq 1$ and where $\Gamma$ is the positively oriented curve $\{z:|z|=1-\delta\}$ where $\delta>0$ is such that $A_{\dagger}(z)$ and $H(z) / H_{\star}(z)$ have all their poles inside the open disk $\{z:|z|<1-\delta\}$.

Proof: The preceding Theorem 5.5 quantifies the asymptotic variability via (93) which involves the reproducing kernel $\varphi_{n}(\lambda, \omega)$ for the space $X_{n}$ spanned by the rows of $\Psi\left(z, \theta_{\circ}^{n}\right)$ defined by (94). Via Lemma 4.2, this space can be expressed as

$$
\begin{array}{r}
X_{n}=\operatorname{Span}\left\{\frac{z^{-1} F(z)}{A^{2}\left(z, \theta_{\circ}^{n}\right) H_{\star}(z)}, \frac{z^{-2} F(z)}{A^{2}\left(z, \theta_{\circ}^{n}\right) H_{\star}(z)}, \ldots,\right. \\
\left.\frac{z^{-\left(m_{a}+m_{b}\right)} F(z)}{A^{2}\left(z, \theta_{\circ}^{n}\right) H_{\star}(z)}\right\} .
\end{array}
$$

However, since (95) holds, then this space can be reformulated as

$$
X_{n}=\operatorname{Span}\left\{\frac{z^{-1}}{A_{\dagger}(z)}, \ldots, \frac{z^{-m_{a}+m_{b}}}{A_{\dagger}(z)}\right\} .
$$

Using this definition of $X_{n}$ in combination with Lemma 3.6 then completes the proof.

The second corollary to Theorem 5.2 provides a more explicit expression for the variability that applies under greater restrictions on the fixed noise model, the input spectrum and the chosen model order.

Corollary 5.6 (Frequency Domain Variability-Fixed MA Noise Model With AR Input): Suppose that all the assumptions of Corollary 5.5 hold. Suppose further that a spectral factor $F(z)$ of the input spectrum $\Phi_{u}(\omega)$ is of the autoregressive form

$$
F(z)=\frac{\mu}{L(z)} \quad L(z)=\prod_{k=1}^{m_{f}}\left(1-f_{k} z^{-1}\right), \quad \mu \in \mathbf{C}
$$

and that $H(z)$ and $H_{\star}(z)$ are both of moving average type

$$
H(z)=\prod_{k=1}^{m_{h}}\left(1-h_{k} z^{-1}\right) \quad H_{\star}(z)=\prod_{k=1}^{m_{h^{\star}}}\left(1-h_{k}^{\star} z^{-1}\right) .
$$

Finally, suppose also that $m_{b}=m_{a}+m_{f}+m_{h^{\star}}+m_{\star}, m_{\star} \geq 0$. Then

$$
\begin{aligned}
& \lim _{N \rightarrow \infty} N \cdot \operatorname{Cov}\left\{G\left(e^{j \omega}, \hat{\theta}_{N}^{n}\right)\right\} \\
& =\frac{\Phi_{\nu}(\omega)}{\Phi_{u}(\omega)} \cdot \kappa(\omega)+2 \frac{\sigma^{2}}{\Phi_{u}(\omega)}\left|H_{\star}\left(e^{j \omega}\right)\right|^{2} \operatorname{Re}\left\{e^{j \omega} \gamma^{\prime}\left(e^{j \omega}\right)\right\}
\end{aligned}
$$


where now

$$
\begin{aligned}
\kappa(\omega)=\left[m_{\star}\right. & +2 \sum_{k=1}^{m_{a}} \frac{1-\left|\xi_{k}\right|^{2}}{\left|e^{j \omega}-\xi_{k}\right|^{2}} \\
& \left.+\sum_{k=1}^{m_{f}} \frac{1-\left|f_{k}\right|^{2}}{\left|e^{j \omega}-f_{k}\right|^{2}}+\sum_{k=1}^{m_{h^{\star}}} \frac{1-\left|h_{k}^{\star}\right|^{2}}{\left|e^{j \omega}-h_{k}^{\star}\right|^{2}}\right]
\end{aligned}
$$

and where $\gamma(z)$ is the positive real part of $H(z) H\left(z^{-1}\right) H_{\star}^{-1}(z) H_{\star}^{-1}\left(z^{-1}\right)$ that is analytic on $\{z:|z|>1\}$.

Proof: Under the assumptions of the corollary, (95) yields

$$
A_{\dagger}(z)=A^{2}\left(z, \theta_{\circ}^{n}\right) H_{\star}(z) L(z)
$$

which is a polynomial of order $2 m_{a}+m_{h_{\star}}+m_{f}$ which, again under the assumptions of the corollary, is of order $m_{a}+m_{b}-m_{\star}$. Consequently, Corollary 5.5 applies with $\kappa(\omega)$ given by (85), which being defined by the zeros of $z^{m_{a}+m_{b}} A_{+}(z)$ is, in this case, defined by the zeros of $A^{2}\left(z, \theta_{\circ}^{n}\right) H_{\star}(z) L(z)$ together with $m_{\star}$ zeros at the origin and, hence, is given by (102). Furthermore, under the MA assumptions on noise models, the poles of $H(z) / H_{\star}(z)$ are a subset, taken without replacement, of the zeros of $z^{m_{a}+m_{b}} A_{\dagger}(z)$ and, hence, by Corollary 3.1, the last term in (96) involving integration around the contour $\Gamma$ is equal to zero.

There are some important remarks to be made in relation to these results.

- Corollary 5.6 extends the results of [10] where, for the case of a model with fixed and correct denominator and then with estimation of only a numerator polynomial, the work there derived an equivalent expression to (101) with the second term replaced by

$$
\begin{aligned}
& -2 \sigma^{2} \frac{\left|H_{\star}\left(e^{j \omega}\right)\right|^{2}}{\Phi_{u}(\omega)} \\
& \quad \times \operatorname{Re}\left\{\frac{1}{2 \pi j} \oint_{|z|=1-\delta} \frac{z e^{j \omega}}{\left(z-e^{j \omega}\right)^{2}}\right. \\
& \left.\quad \times \frac{H(z) H\left(z^{-1}\right)}{H_{\star}(z) H_{\star}\left(z^{-1}\right)} \frac{\mathrm{d} z}{z}\right\} .
\end{aligned}
$$

However, using the positive real decomposition (36) (with $\tilde{H}=H\left(H_{\star}\right)$, the change of variable $z \mapsto z^{-1}$, and recognising that since $\gamma\left(z^{-1}\right)$ is assumed analytic within a disk of radius $(1-\delta)^{-1}$ this term (104) can be rewritten using Cauchy's Residue Theorem as

$$
\begin{aligned}
-2 \sigma^{2} & \frac{\left|H_{\star}\left(e^{j \omega}\right)\right|^{2}}{\Phi_{u}(\omega)} \\
+ & \operatorname{Re}\left\{\frac{1}{2 \pi j} \oint_{|z|=1 /(1-\delta)} \frac{e^{-j \omega}}{\left(z-e^{-j \omega}\right)^{2}} \gamma\left(z^{-1}\right) \mathrm{d} z\right\} \\
= & -2 \sigma^{2} \frac{\left|H_{\star}\left(e^{j \omega}\right)\right|^{2}}{\Phi_{u}(\omega)} \operatorname{Re}\left\{\left.e^{-j \omega} \frac{\mathrm{d}}{\mathrm{d} z} \gamma\left(z^{-1}\right)\right|_{z=e^{-j \omega}}\right\} \\
= & 2 \sigma^{2} \frac{\left|H_{\star}\left(e^{j \omega}\right)\right|^{2}}{\Phi_{u}(\omega)} \operatorname{Re}\left\{e^{j \omega} \gamma^{\prime}\left(e^{j \omega}\right)\right\}
\end{aligned}
$$

which is identical to the last term in our expression (101).
- In relation to this previous comment, suppose that all the conditions of Corollary 5.6 hold, and that furthermore the model structure is restricted to be that of (38) with $G\left(q, \theta^{n}\right)=B\left(q, \theta^{n}\right) / A(q)$ where $A(q)$ is fixed and not dependent on $\theta^{n}$. This is the 'generalized' FIR model (it is exactly FIR if $\left.A(q)=H_{\star}(q)=1\right)$ that [10] studies, and for which the underlying space $X_{n}$ defined by (94) is clearly given as

$$
X_{n}=\operatorname{Span}\left\{\frac{z^{-1}}{A(z) H_{\star}(z) L(z)}, \ldots, \frac{z^{-m_{b}}}{A(z) H_{\star}(z) L(z)}\right\} .
$$

Therefore, provided that the fixed $A(q)$ is equal to the denominator of the true underlying dynamics $G(q)$, and that the numerator order $m_{b}=m_{a}+m_{h_{\star}}+m_{f}+m_{\star} ; m_{\star} \geq 0$, then applying Theorem 5.2 along the lines of the proof of Corollary 5.6 delivers the quantification (101) with

$$
\begin{aligned}
\kappa(\omega)=\left[m_{\star}+\sum_{k=1}^{m_{a}} \frac{1-\left|\xi_{k}\right|^{2}}{\left|e^{j \omega}-\xi_{k}\right|^{2}}\right. & +\sum_{k=1}^{m_{f}} \frac{1-\left|f_{k}\right|^{2}}{\left|e^{j \omega}-f_{k}\right|^{2}} \\
& \left.+\sum_{k=1}^{m_{h^{\star}}} \frac{1-\left|h_{k}^{\star}\right|^{2}}{\left|e^{j \omega}-h_{k}^{\star}\right|^{2}}\right]
\end{aligned}
$$

as being the variance quantification for this model structure. This result is identical to [10, Th. 3.1], and thus the reproducing kernel methods of this paper provide a complementary means for addressing the problems considered in [10].

- The last term in (101) encapsulates the variance increasing effect of a fixed and incorrect noise model. Notice that when $H_{\star} \equiv H$ then the last term in (101) is zero. Furthermore, since $\left|H\left(e^{j \omega}\right) / H_{\star}\left(e^{j \omega}\right)\right|^{2}=2 \operatorname{Re}\left\{\gamma\left(e^{j \omega}\right)\right\}$ the last term in (101) very clearly indicates that the variance increase due to mismatch between $H$ and $H^{\star}$ is directly proportional to the smoothness (as measured by the derivative) of $\left|H / H_{\star}\right|^{2}$.

This observation reinforces remarks in the work [10] which, via an examination of the nature of the integrand component $z e^{j \omega}\left(z-e^{j \omega}\right)^{-2}$ in (104), argues that this term is likely to be large if zeros of $H_{\star}(z)$ are placed near the unit circle, and not cancelled by equivalent zeros in $H(z)$.

In particular, as remarked in [10] in the case of fixed denominator structures, but as now shown here to apply for a much wider class of structures, fixed noise model zeros should only be included when the user is rather sure of their actual existence and location.

- Finally, it is important to note that there are other situations, beyond those stated in Corollary 5.6 for which the exact quantification in Corollary 5.5 holds. For example, when performing indirect identification of closed-loop systems, the noise model and closed-loop dynamics share common poles and, hence, the condition (95) may be satisfied. The consequences of this are taken up in another work [28], but the point is that for certain applications, it is important that Corollaries 5.5 and 5.6 are stated separately. 


\section{CONCLUSION}

This paper has established the new principle that the problem of quantifying variance error is equivalent to that of quantifying the reproducing kernel for a certain subspace of $L_{2}$. Since this subspace was shown to depend on the model structure being employed, this exposes the important result that the variance error is also dependent on the model structure, and this fact is counter to what is suggested by pre-existing quantifications such as (2) which depend on an asymptotic in model order argument.

Furthermore, for certain important special cases, it was shown how the reproducing kernel and, hence, the variance error, could be expressed in closed form, and for a finite model order of interest. These results, since they apply for the very general Box-Jenkins and output-error model structures, are an extension and generalization of previous results that have applied only for FIR-type model structures with fixed denominators.

A point of rapprochement between the new results here and the well known pre-existing one (2) is provided in the companion paper [15] which addresses the case of over-modeling and regularised cost criterion. There, it is established that a choice of regularization point that forces poles at the origin implies a finite-model order variance that approaches the quantification (2) as the model order extends beyond the underlying true one. However, as shown in [15], with different choice of regularization point, this rapprochement is lost.

Another companion paper [28] illustrates how the new expressions derived in this work may be applied to the study of the relative performance of various direct and indirect identification strategies in the context of estimation from data collected under closed-loop conditions, while a further work [29] examines implications of the results here to power spectral density estimation.

Finally, additional work is needed to extend the results here to cover cases of more general input spectrum, and further model classes (such as ARMAX). The authors are currently engaged in the pursuit of these goals.

\section{APPENDIX A \\ PROOF OF LEMMA 3.6}

Proof: Using the result of Lemma 3.3 and noting that $\left|\phi_{n}\left(e^{j \omega}\right)\right|=1$ implies the following:

$$
\begin{aligned}
\varphi_{n} & \left(e^{j \lambda}, e^{j \omega}\right) \varphi_{n}\left(e^{j \omega}, e^{j \lambda}\right) \\
= & \left(\frac{1-\overline{\phi_{n}\left(e^{j \omega}\right)} \phi_{n}\left(e^{j \lambda}\right)}{e^{j(\lambda-\omega)}-1}\right)\left(\frac{1-\overline{\phi_{n}\left(e^{j \lambda}\right)} \phi_{n}\left(e^{j \omega}\right)}{e^{j(\omega-\lambda)}-1}\right) \\
= & 2 \operatorname{Re}\left\{\frac{1-\overline{\phi_{n}\left(e^{j \lambda}\right)} \phi_{n}\left(e^{j \omega}\right)}{\left|e^{j(\lambda-\omega)}-1\right|^{2}}\right\} \\
= & 2 \operatorname{Re}\left\{\frac{\varphi_{n}\left(e^{j \omega}, e^{j \lambda}\right)}{e^{j(\lambda-\omega)}-1} \cdot \frac{e^{j(\omega-\lambda)}}{e^{j(\omega-\lambda)}}\right\} \\
= & 2 \operatorname{Re}\left\{\frac{\varphi_{n}\left(e^{j \omega}, e^{j \omega}\right)}{1-e^{j(\omega-\lambda)}}\right. \\
& \left.+\frac{\varphi_{n}\left(e^{j \omega}, e^{j \lambda}\right) e^{j(\omega-\lambda)}-\varphi_{n}\left(e^{j \omega}, e^{j \omega}\right)}{1-e^{j(\omega-\lambda)}}\right\} \\
= & \varphi_{n}\left(e^{j \omega}, e^{j \omega}\right) \\
& +2 \operatorname{Re}\left\{\frac{\varphi_{n}\left(e^{j \omega}, e^{j \lambda}\right) e^{j(\omega-\lambda)}-\varphi_{n}\left(e^{j \omega}, e^{j \omega}\right)}{1-e^{j(\omega-\lambda)}}\right\} .
\end{aligned}
$$

It is worth noting at this point that the last term in this expression does not possess a singularity at $\lambda=\omega$. Therefore, since $\overline{\varphi_{n}\left(e^{j \lambda}, e^{j \omega}\right)}=\varphi_{n}\left(e^{j \omega}, e^{j \lambda}\right)$

$$
\begin{aligned}
&\left\|\tilde{H}\left(e^{j \lambda}\right) \varphi_{n}\left(e^{j \lambda}, e^{j \omega}\right)\right\|^{2} \\
&= \varphi_{n}\left(e^{j \omega}, e^{j \omega}\right) \cdot \frac{1}{2 \pi} \int_{-\pi}^{\pi}\left|\tilde{H}\left(e^{j \lambda}\right)\right|^{2} \mathrm{~d} \lambda \\
&+2 \operatorname{Re}\left\{\frac{1}{2 \pi} \int_{-\pi}^{\pi} \frac{\varphi_{n}\left(e^{j \omega}, e^{j \lambda}\right) e^{j(\omega-\lambda)}-\varphi_{n}\left(e^{j \omega}, e^{j \omega}\right)}{1-e^{j(\omega-\lambda)}}\right. \\
&\left.\times\left|\tilde{H}\left(e^{j \lambda}\right)\right|^{2} \mathrm{~d} \lambda\right\} .
\end{aligned}
$$

Now, concentrate on the second integral, which can be written in contour form using the positive real decomposition of $\tilde{H}(z) \tilde{H}(1 / z)=\gamma(z)+\gamma\left(z^{-1}\right)$ as

$$
\begin{aligned}
& \frac{1}{2 \pi} \int_{-\pi}^{\pi} \frac{\varphi_{n}\left(e^{j \omega}, e^{j \lambda}\right) e^{j(\omega-\lambda)}-\varphi_{n}\left(e^{j \omega}, e^{j \omega}\right)}{1-e^{j(\omega-\lambda)}} \\
& \quad \times\left[\gamma\left(e^{-j \lambda}\right)+\gamma\left(e^{j \lambda}\right)\right] \mathrm{d} \lambda \\
& =\frac{1}{2 \pi j} \oint_{|z|=1} \frac{\varphi_{n}\left(e^{j \omega}, z\right) z^{-1} e^{j \omega}-\varphi_{n}\left(e^{j \omega}, e^{j \omega}\right)}{1-z^{-1} e^{j \omega}} \\
& \quad \times\left[\gamma\left(z^{-1}\right)+\gamma(z)\right] \frac{\mathrm{d} z}{z} \\
& =\frac{1}{2 \pi j} \oint_{|z|=1-\delta} \frac{\varphi_{n}\left(e^{j \omega}, z\right) z^{-1} e^{j \omega}-\varphi_{n}\left(e^{j \omega}, e^{j \omega}\right)}{z-e^{j \omega}} \gamma(z) \mathrm{d} z .
\end{aligned}
$$

The last equality follows since when $z$ is on the unit circle, then by (27)

$$
\varphi_{n}\left(e^{j \omega}, z\right)=\frac{z\left[1-\overline{\phi_{n}(z)} \phi_{n}\left(e^{j \omega}\right)\right]}{e^{j \omega}-z}
$$

and, hence, the term involving $\gamma\left(z^{-1}\right)$ which vanishes between (A.2) and (A.3) does so since it is analytic within the unit circle. Furthermore, since by the assumptions of the theorem the integrand has no singularities in a neighborhood of the unit circle, then the contour of integration can be changed from $|z|=1$ to $|z|=1-\delta$ for some $\delta>0$ without changing the value of the integral. Furthermore, by the change of variable $z \mapsto z^{-1}$

$$
\begin{aligned}
- & \frac{1}{2 \pi j} \oint_{|z|=1-\delta} \frac{\varphi_{n}\left(e^{j \omega}, e^{j \omega}\right)}{z-e^{j \omega}} \gamma(z) \mathrm{d} z \\
& =\frac{1}{2 \pi j} \oint_{|z|=1 /(1-\delta)} \frac{\varphi_{n}\left(e^{j \omega}, e^{j \omega}\right) e^{-j \omega}}{z-e^{-j \omega}} \gamma\left(z^{-1}\right) \frac{\mathrm{d} z}{z} \\
& =\varphi_{n}\left(e^{j \omega}, e^{j \omega}\right)\left[\gamma\left(e^{j \omega}\right)-\gamma(\infty)\right] .
\end{aligned}
$$

By recognizing that

$$
\begin{aligned}
\frac{1}{2 \pi} \int_{-\pi}^{\pi}\left|\tilde{H}\left(e^{j \lambda}\right)\right|^{2} \mathrm{~d} \lambda & =2 \gamma(\infty) \\
\left|\tilde{H}\left(e^{j \omega}\right)\right|^{2} & =2 \operatorname{Re}\left\{\gamma\left(e^{j \omega}\right)\right\}
\end{aligned}
$$

then, substituting (A.5) into (A.3) and then (A.1) implies that

$$
\begin{aligned}
& \left\|\tilde{H}\left(e^{j \lambda}\right) \varphi_{n}(\lambda, \omega)\right\|^{2}=\varphi_{n}\left(e^{j \omega}, e^{j \omega}\right)\left|\tilde{H}\left(e^{j \omega}\right)\right|^{2} \\
& \quad+2 \operatorname{Re}\left\{\frac{1}{2 \pi j} \oint_{|z|=1-\delta} \frac{\varphi_{n}\left(e^{j \omega}, z\right) z^{-1} e^{j \omega}}{z-e^{j \omega}} \gamma(z) \mathrm{d} z\right\}
\end{aligned}
$$


Furthermore, again using (27)

$$
\begin{aligned}
& \frac{\varphi_{n}\left(e^{j \omega}, z\right) z^{-1} e^{j \omega}}{z-e^{j \omega}} \\
& =\frac{\left[1-\overline{\phi_{n}(z)} \phi_{n}\left(e^{j \omega}\right)\right] z^{-1} e^{j \omega}}{\left(z-e^{j \omega}\right)\left(z^{-1} e^{j \omega}-1\right)} \\
& =\frac{-e^{j \omega}}{\left(z-e^{j \omega}\right)^{2}}+\frac{e^{j \omega} \overline{\phi_{n}(z)} \phi_{n}\left(e^{j \omega}\right)}{\left(z-e^{j \omega}\right)^{2}} .
\end{aligned}
$$

Now using the change of variable $z \mapsto z^{-1}$

$$
\begin{aligned}
& \frac{1}{2 \pi j} \oint_{|z|=1-\delta} \frac{1}{\left(z-e^{j \omega}\right)^{2}} \gamma(z) \mathrm{d} z \\
& \quad=\frac{1}{2 \pi j} \oint_{|z|=1 /(1-\delta)} \frac{e^{-j 2 \omega}}{\left(z-e^{-j \omega}\right)^{2}} \gamma\left(z^{-1}\right) \mathrm{d} z \\
& \quad=\left.e^{-j 2 \omega} \frac{\mathrm{d}}{\mathrm{d} z} \gamma\left(z^{-1}\right)\right|_{z=e^{-j \omega}}=-\gamma^{\prime}\left(e^{j \omega}\right) .
\end{aligned}
$$

Therefore, using (A.9) and (A.8) in (A.7) leads to

$$
\begin{aligned}
& \left\|\tilde{H}\left(e^{j \lambda}\right) \varphi_{n}(\lambda, \omega)\right\|^{2} \\
& =\varphi_{n}\left(e^{j \omega}, e^{j \omega}\right)\left|\tilde{H}\left(e^{j \omega}\right)\right|^{2}+2 \operatorname{Re}\left\{e^{j \omega} \gamma^{\prime}\left(e^{j \omega}\right)\right\} \\
& \quad+2 \operatorname{Re}\left\{\frac{1}{2 \pi j} \oint_{|z|=1-\delta} \frac{e^{j \omega} \overline{\phi_{n}(z)} \phi_{n}\left(e^{j \omega}\right)}{\left(z-e^{j \omega}\right)^{2}} \gamma(z) \mathrm{d} z\right\} .
\end{aligned}
$$

\section{APPENDIX B}

\section{SPACES INDUCED By MODEL STRUCTURES}

\section{A. Proof of Lemma 4.1}

Proof: First, according to (40)

$$
\begin{aligned}
\psi_{t}\left(\theta^{n}\right)= & -\frac{\mathrm{d}}{\mathrm{d} \theta^{n}} \hat{y}_{t}(\theta) \\
= & H^{-1}\left(q, \theta^{n}\right) \frac{\mathrm{d} G\left(q, \theta^{n}\right)}{\mathrm{d} \theta^{n}} u_{t} \\
& -H^{-2}\left(q, \theta^{n}\right) \frac{\mathrm{d} H\left(q, \theta^{n}\right)}{\mathrm{d} \theta^{n}} G\left(q, \theta^{n}\right) u_{t} \\
& +H^{-2}\left(q, \theta^{n}\right) \frac{\mathrm{d} H\left(q, \theta^{n}\right)}{\mathrm{d} \theta^{n}} y_{t} .
\end{aligned}
$$

Substituting (41) into (B.1) then establishes that (55) satisfies (48). Furthermore, since for any $k$ in the appropriate range

$$
\begin{aligned}
\frac{\mathrm{d}}{\mathrm{d} a_{k}} G\left(z, \theta^{n}\right) & =-\frac{G\left(z, \theta^{n}\right) z^{-k}}{A\left(z, \theta^{n}\right)} \\
\frac{\mathrm{d}}{\mathrm{d} b_{k}} G\left(z, \theta^{n}\right) & =\frac{z^{-k}}{A\left(z, \theta^{n}\right)} \\
\frac{\mathrm{d} H\left(z, \theta^{n}\right)}{\mathrm{d} d_{k}} & =-\frac{H\left(z, \theta^{n}\right) z^{-k}}{D\left(z, \theta^{n}\right)} \\
\frac{\mathrm{d} H\left(z, \theta^{n}\right)}{\mathrm{d} c_{k}} & =\frac{z^{-k}}{D\left(z, \theta^{n}\right)} .
\end{aligned}
$$

and

$$
\begin{aligned}
\frac{\mathrm{d}}{\mathrm{d} c_{k}} G\left(z, \theta^{n}\right) & =\frac{\mathrm{d}}{\mathrm{d} d_{k}} G\left(z, \theta^{n}\right)=\frac{\mathrm{d}}{\mathrm{d} a_{k}} H\left(z, \theta^{n}\right) \\
& =\frac{\mathrm{d}}{\mathrm{d} b_{k}} H\left(z, \theta^{n}\right)=0 .
\end{aligned}
$$

Therefore, since under the assumption of $\Phi_{u e} \equiv 0, \Phi_{u}(\omega)=$ $\left|F\left(e^{j \omega}\right)\right|^{2}$

$$
S_{\zeta}(z)=\left[\begin{array}{cc}
F(z) & 0 \\
0 & \sigma
\end{array}\right]
$$

and then according to (50) and (49)

$$
\begin{aligned}
X_{n}= & \operatorname{span}\left\{\left[\frac{G\left(z, \theta_{\circ}^{n}\right) F(z) z^{-1}}{H\left(z, \theta_{\circ}^{n}\right) A\left(z, \theta_{\circ}^{n}\right)}, 0\right]^{T}, \ldots,\right. \\
& {\left.\left[\frac{G\left(z, \theta_{\circ}^{n}\right) F(z) z^{-m_{a}}}{H\left(z, \theta_{\circ}^{n}\right) A\left(z, \theta_{\circ}^{n}\right)}, 0\right]^{T}\right\} } \\
\oplus & \operatorname{Span}\left\{\left[\frac{F(z) z^{-1}}{H\left(z, \theta_{\circ}^{n}\right) A\left(z, \theta_{\circ}^{n}\right)}, 0\right]^{T}, \ldots,\right. \\
& {\left.\left[\frac{F(z) z^{-m_{b}}}{H\left(z, \theta_{\circ}^{n}\right) A\left(z, \theta_{\circ}^{n}\right)}, 0\right]^{T}\right\} } \\
\oplus & \operatorname{Span}\left\{\left[0, \frac{z^{-1}}{D\left(z, \theta_{\circ}^{n}\right)}\right]^{T}, \ldots,\left[0, \frac{z^{-m_{d}}}{D\left(z, \theta_{\circ}^{n}\right)}\right]^{T},\right. \\
& {\left.\left[0, \frac{z^{-1}}{C\left(z, \theta_{\circ}^{n}\right)}\right]^{T}, \ldots,\left[0, \frac{z^{-m_{c}}}{C\left(z, \theta_{\circ}^{n}\right)}\right]^{T}\right\} }
\end{aligned}
$$

which can be alternatively expressed as (56) and (57).

\section{B. Proof of Lemma 4.2}

Proof: Since $H\left(q, \theta^{n}\right)=1$ in this case, then $\mathrm{d} H\left(q, \theta^{n}\right) / \mathrm{d} \theta^{n}=0$. Substituting this into (B.1) then establishes that (59) satisfies (48). Furthermore, the expression (B.2) holds again in this case, and therefore, the space $X_{n}$ is given as

$$
\begin{gathered}
X_{n}=\operatorname{Span}\left\{\frac{G\left(z, \theta_{\circ}^{n}\right) F(z) z^{-1}}{A\left(z, \theta_{\circ}^{n}\right)}, \ldots, \frac{G\left(z, \theta_{\circ}^{n}\right) F(z) z^{-m_{a}}}{A\left(z, \theta_{\circ}^{n}\right)},\right. \\
\left.\frac{F(z) z^{-1}}{A\left(z, \theta_{\circ}^{n}\right)}, \ldots, \frac{F(z) z^{-m_{b}}}{A\left(z, \theta_{\circ}^{n}\right)}\right\}
\end{gathered}
$$

which can be alternatively expressed as (60).

\section{Proof of Lemma 4.3}

Proof: The expression (B.2) hold again in this case, and furthermore, for any $k$

$$
\begin{aligned}
\frac{\mathrm{d}}{\mathrm{d} c_{k}} G\left(q, \theta^{n}\right) & =\frac{\mathrm{d}}{\mathrm{d} b_{k}} H\left(q, \theta^{n}\right)=0 \\
\frac{\mathrm{d}}{\mathrm{d} a_{k}} H(q, \theta) & =-\frac{H(q, \theta)}{A(q, \theta)} \cdot q^{-k} \\
\frac{\mathrm{d}}{\mathrm{d} c_{k}} H(q, \theta) & =\frac{q^{-k}}{A(q, \theta)} .
\end{aligned}
$$

Therefore, since (B.5) again holds, then according to (50) and (49)

$$
\begin{gathered}
X_{n}=\operatorname{Span}\left\{\left[\frac{G\left(z, \theta_{\circ}^{n}\right) F(z) z^{-1}}{C\left(z, \theta_{\circ}^{n}\right)}, \frac{z^{-1}}{A\left(z, \theta_{\circ}^{n}\right)}\right]^{T}, \ldots,\right. \\
\left.\left[\frac{G\left(z, \theta_{\circ}^{n}\right) F(z) z^{-m_{a}}}{C\left(z, \theta_{\circ}^{n}\right)}, \frac{z^{-m_{a}}}{A\left(z, \theta_{\circ}^{n}\right)}\right]^{T}\right\}
\end{gathered}
$$




$$
\begin{aligned}
& \oplus \operatorname{Span}\left\{\left[\frac{F(z) z^{-1}}{C\left(z, \theta_{\circ}^{n}\right)}, 0\right]^{T}, \ldots,\left[\frac{F(z) z^{-m_{b}}}{C\left(z, \theta_{\circ}^{n}\right)}, 0\right]^{T}\right\} \\
& \oplus \operatorname{Span}\left\{\left[0, \frac{z^{-1}}{C\left(z, \theta_{\circ}^{n}\right)}\right]^{T}, \ldots,\left[0, \frac{z^{-m_{c}}}{C\left(z, \theta_{\circ}^{n}\right)}\right]^{T}\right\}
\end{aligned}
$$

which can be alternatively expressed as (64) and (57).

\section{APPENDIX C \\ PROOF OF THEOREM 5.1}

Proof: From [2], we have that the parameter covariance matrix $P_{n}$ in (45) is defined in terms of two other matrices $R_{n}$ and $Q_{n}$ as

$$
P_{n} \triangleq R_{n}^{-1} Q_{n} R_{n}^{-1}
$$

which themselves are specified as

$$
\begin{aligned}
R_{n} \triangleq & \lim _{N \rightarrow \infty} \frac{1}{N} \sum_{t=1}^{N}\left[\mathrm{E}\left\{\psi_{t}\left(\theta_{\circ}^{n}\right) \psi_{t}^{T}\left(\theta_{\circ}^{n}\right)\right\}\right. \\
& \left.-\mathrm{E}\left\{\varepsilon_{t}\left(\theta_{\circ}^{n}\right)\left(\frac{\mathrm{d} \psi_{t}\left(\theta^{n}\right)}{\mathrm{d} \theta^{n}}\right)^{T}\right\}\right]
\end{aligned}
$$

and

$$
\begin{aligned}
Q_{n} \triangleq & \lim _{N \rightarrow \infty} \frac{1}{N} \sum_{t=1}^{N} \sum_{\ell=1}^{N} \mathrm{E}\left\{\psi_{t}\left(\theta_{\circ}^{n}\right) \psi_{\ell}^{T}\left(\theta_{\circ}^{n}\right)\right. \\
& \left.\times \varepsilon_{t}\left(\theta_{\circ}^{n}\right) \varepsilon_{\ell}\left(\theta_{\circ}^{n}\right)\right\} .
\end{aligned}
$$

The quantity $\psi_{t}\left(\theta^{n}\right)$ in the preceding expressions is the prediction error gradient given by (48). Therefore, under the assumptions of the theorem that $\varepsilon_{t}\left(\theta_{\circ}^{n}\right)=e_{t}$ and using Parseval's Theorem

$$
\begin{aligned}
R_{n} & =\lim _{N \rightarrow \infty} \frac{1}{N} \sum_{t=1}^{N} \mathrm{E}\left\{\psi_{t}\left(\theta_{\circ}^{n}\right) \psi_{t}^{T}\left(\theta_{\circ}^{n}\right)\right\} \\
& =\frac{1}{2 \pi} \int_{-\pi}^{\pi} \Psi\left(e^{j \omega}, \theta_{\circ}^{n}\right) \Psi^{\star}\left(e^{j \omega}, \theta_{\circ}^{n}\right) \mathrm{d} \omega \\
Q_{n} & \triangleq \lim _{N \rightarrow \infty} \frac{\sigma^{2}}{N} \sum_{t=1}^{N} \mathrm{E}\left\{\psi_{t}\left(\theta_{\circ}^{n}\right) \psi_{t}^{T}\left(\theta_{\circ}^{n}\right)\right\}=\sigma^{2} R_{n}
\end{aligned}
$$

where $\Psi\left(z, \theta^{n}\right)$ is defined in (49). Therefore, the matrix $P_{n}$ quantifying the parameter space variability of $\hat{\theta}_{N}^{n}$ is given as

$$
P_{n}=\sigma^{2} R_{n}^{-1}
$$

where the inverse is guaranteed to exist by the assumptions of the theorem and Lemma E.1. Furthermore, according to a first-order Taylor expansion, the relationship between frequency domain and parameter space estimation errors is given as

$$
\begin{aligned}
\Pi^{T}\left(e^{j \omega}, \hat{\theta}_{N}^{n}\right)-\Pi^{T}\left(e^{j \omega}, \theta_{\circ}^{n}\right) \\
=\left[\left.\frac{\mathrm{d} \Pi\left(e^{j \omega}, \theta^{n}\right)}{\mathrm{d} \theta^{n}}\right|_{\theta^{n}=\theta_{\circ}^{n}}\right]^{T}\left(\hat{\theta}_{N}^{n}-\theta_{\circ}^{n}\right) \\
\quad+o\left(\left\|\hat{\theta}_{N}^{n}-\theta_{\circ}^{n}\right\|^{2}\right) .
\end{aligned}
$$

Therefore, a consequence of (45) is that

$$
\begin{aligned}
& \sqrt{N}\left[\begin{array}{c}
G\left(e^{j \omega}, \hat{\theta}_{N}^{n}\right)-G\left(e^{j \omega}, \theta_{\circ}^{n}\right) \\
H\left(e^{j \omega}, \hat{\theta}_{N}^{n}\right)-H\left(e^{j \omega}, \theta_{\circ}^{n}\right)
\end{array}\right] \\
& \stackrel{\mathcal{D}}{\rightarrow} \mathcal{N}\left(0, \sigma^{2} \Delta_{n}(\omega)\right) \quad \text { as } N \rightarrow \infty
\end{aligned}
$$

where

$$
\begin{aligned}
& \Delta_{n}(\omega) \triangleq \frac{1}{\sigma^{2}}\left[\left.\frac{\mathrm{d} \Pi\left(e^{j \omega}, \theta^{n}\right)}{\mathrm{d} \theta^{n}}\right|_{\theta^{n}=\theta_{\circ}^{n}}\right]^{T} \\
& \quad \times P_{n}\left[\left.\frac{\mathrm{d} \Pi\left(e^{-j \omega}, \theta^{n}\right)}{\mathrm{d} \theta^{n}}\right|_{\theta^{n}=\theta_{\circ}^{n}}\right] .
\end{aligned}
$$

Furthermore, according to (49)

$$
\begin{aligned}
{\left[\frac{\mathrm{d} \Pi\left(e^{j \omega}, \theta^{n}\right)}{\mathrm{d} \theta^{n}} \mid\right.} & \theta_{\theta^{n}=\theta_{\circ}^{n}} \\
& =H\left(e^{j \omega}, \theta_{\circ}^{n}\right) \Psi\left(e^{j \omega}, \theta_{\circ}^{n}\right) S_{\zeta_{\circ}}^{-1}\left(e^{j \omega}\right) .
\end{aligned}
$$

This expression and (C.6), substituted into (C.9), implies

$$
\begin{aligned}
\Delta_{n}(\omega)=\sigma^{2} \mid H & \left.\left(e^{j \omega}, \theta_{\circ}^{n}\right)\right|^{2} S_{\zeta_{\circ}}^{-\star}\left(e^{j \omega}\right) \Psi^{\star} \\
& \times\left(e^{j \omega}, \theta_{\circ}^{n}\right) R_{n}^{-1} \Psi\left(e^{j \omega}, \theta_{\circ}^{n}\right) S_{\zeta_{\circ}}^{-1}\left(e^{j \omega}\right)
\end{aligned}
$$

Finally, by Lemma $3.2 \Psi^{\star}\left(e^{j \omega}, \theta_{\circ}^{n}\right) R_{n}^{-1} \Psi\left(e^{j \lambda}, \theta_{\circ}^{n}\right)=$ $\varphi_{n}(\lambda, \omega)$, which is the reproducing kernel for the subspace spanned by the rows of $\Psi\left(z, \theta_{0}^{n}\right)$, which completes the proof.

\section{APPENDIX D}

\section{PROOF OF THEOREM 5.2}

Proof: Under the conditions of the Theorem $\zeta_{t}\left(\theta_{\circ}^{n}\right)=u_{t}$ and, hence, according to (49) and with $F(z)$ being the spectral factor of $\Phi_{u}$

$$
\Psi\left(z, \theta_{\circ}^{n}\right)=\left.H_{\star}^{-1}(z) \frac{\mathrm{d} G\left(z, \theta^{n}\right)}{\mathrm{d} \theta^{n}}\right|_{\theta^{n}=\theta_{\circ}^{n}} F(z) .
$$

Furthermore, the parameter space covariance matrix $P_{n}$ is again given as (C.1), but since now $\mathcal{S} \notin \mathcal{M}$ is assumed so that $\varepsilon_{t}\left(\theta_{o}^{n}\right) \neq e_{t}$, then using the same Taylor expansion argument as used in the previous Appendix C (where $\mathrm{d} G\left(z, \theta^{n}\right) / \mathrm{d} \theta^{n}$ is expressed via (D.1)) the asymptotic in $N$ frequency domain variability is given by

$$
\begin{aligned}
N & \cdot \operatorname{Cov}\left\{G\left(e^{j \omega}, \hat{\theta}_{N}^{n}\right)\right\} \\
& =\sigma^{2}\left|\frac{H_{\star}\left(e^{j \omega}\right)}{F\left(e^{j \omega}\right)}\right|^{2} \Psi^{\star}\left(e^{j \omega}, \theta_{\circ}^{n}\right) R_{n}^{-1} Q_{n} R_{n}^{-1} \Psi\left(e^{j \omega}, \theta_{\circ}^{n}\right) .
\end{aligned}
$$

Now, note that under the assumptions of the lemma

$$
\varepsilon_{t}\left(\theta_{\circ}^{n}\right)=H_{\star}^{-1}(q) H(q) e_{t}
$$

so that under the assumption of $\Phi_{u e}(\omega)=0$ then

$$
\mathrm{E}\left\{\varepsilon_{t}\left(\theta_{\circ}^{n}\right)\left(\frac{\mathrm{d} \psi_{t}\left(\theta^{n}\right)}{\mathrm{d} \theta^{n}}\right)^{T}\right\}=0
$$


and, hence, $R_{n}$ is given by (D.4). Also, since by assumption $G(q)=G\left(q, \theta_{\circ}^{n}\right)$, then $\varepsilon_{t}\left(\theta_{\circ}^{n}\right)$ is uncorrelated with $\psi_{t}\left(\theta_{\circ}^{n}\right)$ and, therefore, by [14, Lemma A.1]

$$
Q_{n}=\frac{\sigma^{2}}{2 \pi} \int_{-\pi}^{\pi} \Psi\left(e^{j \lambda}, \theta_{\circ}^{n}\right) \Psi^{\star}\left(e^{j \lambda}, \theta_{\circ}^{n}\right)\left|\frac{H\left(e^{j \lambda}\right)}{H_{\star}\left(e^{j \lambda}\right)}\right|^{2} \mathrm{~d} \lambda .
$$

An application of Lemma 3.5 then implies that

$$
\begin{aligned}
\Psi^{\star}\left(e^{j \omega}, \theta_{\circ}^{n}\right) R_{n}^{-1} Q_{n} R_{n}^{-1} \Psi & \left(e^{j \omega}, \theta_{\circ}^{n}\right) \\
& =\left\|\frac{H\left(e^{j \lambda}\right)}{H_{\star}\left(e^{j \lambda}\right)} \varphi_{n}(\lambda, \omega)\right\|^{2}
\end{aligned}
$$

where $\varphi_{n}(\lambda, \omega)$ is the reproducing kernel for the subspace spanned by the rows of $\Psi\left(z, \theta_{\circ}^{n}\right)$ defined in (D.1).

\section{APPENDIX E \\ TECHNICAL LEMMA}

Lemma E.1: Suppose that the spectral density matrix $\Phi_{\zeta}(\omega)>0$ for all $\omega \in[-\pi, \pi]$, and that with $\Psi\left(z, \theta^{n}\right)$ being defined in (49), the conditions of Lemma 4.1 hold with the model structure (38) being employed having dynamics model $G\left(q, \theta^{n}\right)$ and noise model $H\left(q, \theta^{n}\right)$ independently parametrized. Then, the matrix

$$
T_{n}=\frac{1}{2 \pi} \int_{-\pi}^{\pi} \Psi\left(e^{j \omega}, \theta_{\circ}^{n}\right) \Psi^{\star}\left(e^{j \omega}, \theta_{\circ}^{n}\right) \mathrm{d} \omega
$$

is positive definite and, hence, invertible, if and only if there are no pole zero cancellations in both of $G\left(z, \theta_{\circ}^{n}\right)$ and $H\left(z, \theta_{\circ}^{n}\right)$.

Proof: Suppose that the matrix (E.1) is rank deficient. Then by the definition (49) there exists a nonzero $x \in \mathbf{C}^{n}$ such that

$$
\begin{aligned}
0= & \frac{1}{2 \pi} \int_{-\pi}^{\pi}\left[x^{\star} \frac{\mathrm{d} G\left(e^{j \omega}, \theta_{\circ}^{n}\right)}{\mathrm{d} \theta^{n}}, x^{\star} \frac{\mathrm{d} H\left(e^{j \omega}, \theta_{\circ}^{n}\right)}{\mathrm{d} \theta^{n}}\right] \\
& \times \frac{\Phi_{\zeta}(\omega)}{\left|H\left(e^{j \omega}, \theta_{\circ}^{n}\right)\right|^{2}}\left[x^{\star} \frac{\mathrm{d} G\left(e^{j \omega}, \theta_{\circ}^{n}\right)}{\mathrm{d} \theta^{n}}, x^{\star} \frac{\mathrm{d} H\left(e^{j \omega}, \theta_{\circ}^{n}\right)}{\mathrm{d} \theta^{n}}\right]^{\star} \mathrm{d} \omega .
\end{aligned}
$$

Now, since $\Phi_{\zeta}(\omega)>0$ for all $\omega \in[-\pi, \pi]$, then the aforementioned integrand is strictly nonnegative. Therefore, the integral is equal to zero if, and only if

$$
x^{\star} \frac{\mathrm{d} G\left(e^{j \omega}, \theta_{\circ}^{n}\right)}{\mathrm{d} \theta^{n}}=0 \text { and } x^{\star} \frac{\mathrm{d} H\left(e^{j \omega}, \theta_{\circ}^{n}\right)}{\mathrm{d} \theta^{n}}=0
$$

for all $\omega \in[-\pi, \pi]$. Now, consider the first equality concerning $G\left(z, \theta_{\circ}^{n}\right)$. First

$$
\begin{aligned}
x^{\star} \frac{\mathrm{d} G\left(z, \theta_{\circ}^{n}\right)}{\mathrm{d} \theta^{n}}=\frac{1}{A^{2}\left(z, \theta_{\circ}^{n}\right)}\left[p_{m_{b}}(z) A\left(z, \theta_{\circ}^{n}\right)\right. & \\
& \left.+p_{m_{a}}(z) B\left(z, \theta_{\circ}^{n}\right)\right]
\end{aligned}
$$

for some polynomials $p_{m_{a}}(z), p_{m_{b}}(z)$ of the form

$$
\begin{aligned}
p_{m_{b}}(z)=\beta_{1} z^{-1}+\beta_{2} z^{-2}+\cdots+\beta_{m_{b}} z^{-m_{b}} \\
p_{m_{a}}(z)=\alpha_{1} z^{-1}+\cdots+\alpha_{m_{a}} z^{-m_{a}} .
\end{aligned}
$$

Furthermore, since the lowest powers of $z^{-1}$ in $A\left(z, \theta^{n}\right), B\left(z, \theta^{n}\right)$ are $z^{0}$ and $z^{-1}$, respectively, then

$$
p_{m_{b}}(z) A\left(z, \theta_{\circ}^{n}\right)+p_{m_{a}}(z) B\left(z, \theta_{\circ}^{n}\right)=0
$$

is only possible if the co-efficient of the $z^{-1}$ term $\beta_{1}$ in $p_{m_{b}}(z)$ is set to zero. In this case, there are $m_{a}+m_{b}-1$ degrees of freedom remaining in the choice of the coefficients of $p_{m_{a}}(z)$ and $p_{m_{b}}(z)$ to satisfy (E.5), which may be expressed as

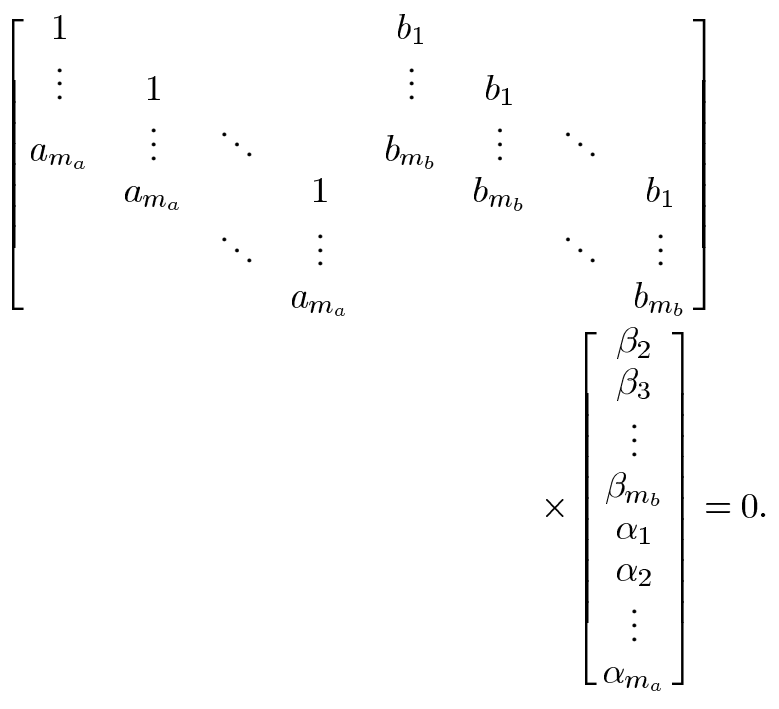

The determinant of the previous matrix is known as the Sylvester resultant, which is guaranteed nonzero provided that $A\left(z, \theta_{\circ}^{n}\right)$ and $B\left(z, \theta_{\circ}^{n}\right)$ are relatively prime [30], in which case the only solution for (E.6) and, hence, the left-hand side of (E.2), is the zero one.

On the other hand, if there are $\ell$ nontrivial common factors between $A\left(z, \theta_{\circ}^{n}\right)$ and $B\left(z, \theta_{\circ}^{n}\right)$, then (E.5) represents $m_{a}+m_{b}-$ $1-\ell$ equality constraints, but with $m_{a}+m_{b}-1$ degrees of freedom in the choice of $p_{m_{a}}(z)$ and $p_{m_{b}}(z)$. Consequently, if $\ell>0$, then a nonzero $p_{m_{a}}(z)$ and/or $p_{m_{b}}(z)$ exists that satisfies (E.5).

Via an identical argument, the right hand side equality is achievable for some nonzero vector $x$ if, and only if there are pole-zero cancellations in $H\left(z, \theta_{\circ}^{n}\right)$. Furthermore, if the noise model $H\left(q, \theta^{n}\right)$ is parametrized independently of $G\left(q, \theta^{n}\right)$, then nonzero rows of $\mathrm{d} G\left(q, \theta^{n}\right) / \mathrm{d} \theta^{n}$ correspond to zero rows of $\mathrm{d} H\left(q, \theta^{n}\right) / \mathrm{d} \theta^{n}$ and vice versa. Therefore, the matrix (E.1) is positive definite if, and only if, there are no pole zero cancellations in both of $G\left(z, \theta_{\circ}^{n}\right)$ and $H\left(z, \theta_{\circ}^{n}\right)$.

\section{REFERENCES}

[1] L. Ljung and L. Guo, "The role of model validation for assessing the size of the unmodeled dynamics," IEEE Trans. Automat. Contr., vol. 42, pp. 1230-1240, Aug. 1997.

[2] L. Ljung, System Identification: Theory for the User, 2nd ed. Upper Saddle River, NJ: Prentice-Hall, 1999.

[3] T. Söderström and P. Stoica, System Identification. New York: Prentice-Hall, 1989.

[4] L. Ljung, "Asymptotic variance expressions for identified black-box transfer function models," IEEE Trans. Automat. Contr., vol. AC-30, pp. 834-844, Sept. 1985. 
[5] L. Ljung and Z. D. Yuan, "Asymptotic properties of black-box identification of transfer functions," IEEE Trans. Automat. Contr., vol. AC-30, pp. 514-530, June 1985.

[6] L. Ljung and B. Wahlberg, "Asymptotic properties of the least squares method for estimating transfer functions and disturbance spectra," $A d v$. Appl. Probab., vol. 24, pp. 412-440, 1992.

[7] D. R. Brillinger, Time Series: Data Analysis and Theory. San Francisco, CA: Holden-Day, 1981.

[8] M. Gevers, L. Ljung, and P. Van den Hof, "Asymptotic variance expressions for closed loop identification," Automatica, vol. 37, pp. 781-786, 2001

[9] P. Van den Hof, "Closed-loop issues in system identification," Annu. Rev. Control, vol. 22, pp. 173-186, 1998

[10] L.-L. Xie and L. Ljung, "Asymptotic variance expressions for estimated frequency functions," IEEE Trans. Automat. Contr., vol. 46, pp. 1887-1899, Dec. 2001.

[11] B. Wahlberg, "System identification using Laguerre models," IEEE Trans. Automat. Contr., vol. 36, pp. 551-562, May 1991.

[12] —, "System identification using Kautz models," IEEE Trans. Automat. Contr., vol. 39, pp. 1276-1282, June 1994.

[13] P. M. J. Van den Hof, P. S. C. Heuberger, and J. Bokor, "System identification with generalized orthonormal basis functions," Automatica, vol. 31, no. 12, pp. 1821-1834, Dec. 1995.

[14] B. Ninness, H. Hjalmarsson, and F. Gustafsson, "The fundamental role of general orthonormal bases in system identification," IEEE Trans. Automat. Contr., vol. 44, pp. 1384-1406, July 1999.

[15] B. Ninness and H. Hjalmarsson, "The effect of regularization on variance error," IEEE Trans. Automat. Contr., vol. 49, pp. 1142-1147, July 2004

[16] B. Epstein, Orthogonal Families of Analytic Functions. New York: Macmillan, 1965.

[17] D. Alpay, The Schur Algorithm, Reproducing Kernel Spaces, and System Theory. Providence, RI: AMS, 1998.

[18] F. Riesz and B. Sz.-Nagy, Functional Analysis. New York: Fredrick Ungar, 1955.

[19] B. Ninness and F. Gustafsson, "A unifying construction of orthonormal bases for system identification," IEEE Trans. Automat. Contr., vol. 42, pp. 515-521, Apr. 1997.

[20] U. Grenander and G. Szegö, Toeplitz Forms and Their Applications. Berkeley, CA: Univ. California Press, 1958.

[21] B. Ninness, H. Hjalmarsson, and F. Gustafsson, "Generalized Fourier and Toeplitz results for rational orthonormal bases," SIAM J. Control Optim., vol. 37, no. 2, pp. 429-460, 1999.

[22] P. Caines, Linear Stochastic Systems. New York: Wiley, 1988.

[23] L. Ljung, MATLAB System Identification Toolbox Users Guide, Version 5. Natick, MA: The Mathworks, 2000.

[24] P. Stoica and R. Moses, Introduction to Spectral Analysis. Upper Saddle River, NJ: Prentice-Hall, 1997.

[25] L. Ljung, "Convergence analysis of parametric identification methods," IEEE Trans. Automat. Contr., vol. AC-23, pp. 770-783, May 1978.
[26] L. Ljung and P. E. Caines, "Asymptotic normality of prediction error estimators for approximate system models," Stochastics, vol. 3, pp. 29-46, 1979.

[27] E. Hannan and D. Nicholls, "The estimation of the prediction error variance," J. Amer. Statist. Assoc., pt. 1, vol. 72, no. 360, pp. 834-840, 1977.

[28] B. Ninness and H. Hjalmarsson, "On the frequency domain accuracy of closed loop estimates," Automatica, 2004, submitted for publication.

[29] B. Ninness, "The asymptotic CRLB for the spectrum of ARMA processes,” IEEE Trans. Signal Processing, vol. 51, pp. 1520-1531, June 2003.

[30] T. Kailath, Linear Systems. Upper Saddle River, NJ: Prentice-Hall, 1980.

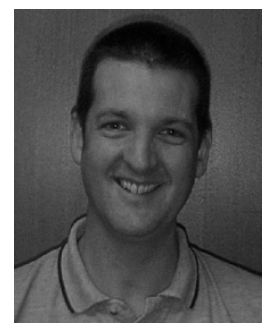

Brett Ninness was born in 1963 in Singleton, Australia. He received the B.E., M.E., and Ph.D. degrees in electrical engineering from the University of Newcastle, Newcastle, Australia, in 1986, 1991, and 1994 , respectively.

Since 1993, he has been with the School of Electrical Engineering and Computer Science at the University of Newcastle, where he is currently an Associate Professor. Details of his research and teaching activities are available at www.ee.newcastle.edu.au/brett.

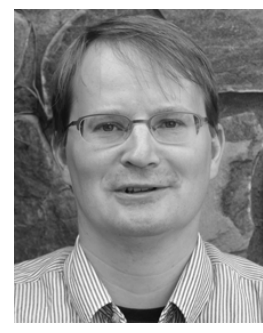

Häkan Hjalmarsson was born in 1962. He received the M.S. degree in electrical engineering in 1988 , and the Licentiate and Ph.D. degrees, both from Linköping University, Sweden, in automatic control in 1990 and 1993, respectively.

$\mathrm{He}$ has held visiting research positions at the California Institute of Technology, Pasadena, Louvain University, Belgium, and the University of Newcastle, Newcastle, Australia. He is a Professor in the Department of Signals, Sensors, and Systems at the Royal Institute of Technology (KTH), Stockholm, Sweden. He has more than 100 publications in the areas of system identification, signal processing, control and estimation in communication networks, and automated tuning of controllers.

Dr. Hjalmarsson was Associate Editor for Automatica between 1996-2002 and has been Guest Editor for the European Journal of Control and Control Engineering Practice. He has been a Member of the IFAC Technical Committee on Modeling, Identification and Signal Processing since 1996 and is co-Chair for SYSID 2006. In 2001, he received the KTH award for outstanding contribution to undergraduate education. 